8-1988

\title{
Effect of Closed Classical Orbits on Quantum Spectra: Ionization of Atoms in a Magnetic Field. I. Physical Picture and Calculations
}

M. L. Du

John B. Delos

William \& Mary, jbdelos@wm.edu

Follow this and additional works at: https://scholarworks.wm.edu/aspubs

Part of the Physics Commons

\section{Recommended Citation}

Du, M. L. and Delos, John B., Effect of Closed Classical Orbits on Quantum Spectra: Ionization of Atoms in a Magnetic Field. I. Physical Picture and Calculations (1988). Physical Review A, 38(4), 1896-1912. https://doi.org/10.1103/PhysRevA.38.1896

This Article is brought to you for free and open access by the Arts and Sciences at W\&M ScholarWorks. It has been accepted for inclusion in Arts \& Sciences Articles by an authorized administrator of W\&M ScholarWorks. For more information, please contact scholarworks@wm.edu. 


\title{
Effect of closed classical orbits on quantum spectra: Ionization of atoms in a magnetic field. I. Physical picture and calculations
}

\author{
M. L. Du and J. B. Delos \\ Joint Institute for Laboratory Astrophysics, University of Colorado and National Bureau of Standards, \\ Boulder, Colorado 80309-0440 \\ and Department of Physics, College of William and Mary, Williamsburg, Virginia 23185*
}

(Received 30 November 1987; revised manuscript received 15 April 1988)

This is the first of two papers that develop the theory of oscillatory spectra. When an atom is placed in a magnetic field, and the absorption spectrum into states close to the ionization threshold is measured at finite resolution, so that individual energy levels are not resolved, it is found that the absorption as a function of energy is a superposition of sinusoidal oscillations. These papers present a quantitative theory of this phenomenon. In this first paper, we describe the physical ideas underlying the theory in the simplest possible way, and we present our first calculations based upon the theory. In the second paper, the theory is developed in full detail, proofs of all of the assertions are given, and we describe the algorithm that was used to make the calculations.

\section{HYDROGEN ATOM IN A MAGNETIC FIELD}

\section{A. Coordinates and Hamiltonian}

For any one-electron atom placed in a uniform magnetic field pointing in the positive $z$ direction, the motion of the electron relative to the nucleus is usually described in a frame of reference that precesses about the $z$ axis at the Larmor frequency. ${ }^{1}$ Coordinates for the electron are generally denoted $\mathbf{q}$; usually cylindrical or spherical coordinates are used, so that $\mathbf{q} \leftrightarrow(\rho, z, \phi)$ or $(r, \theta, \phi)$. In the precessing frame the Hamiltonian is

$$
H=\frac{1}{2 m_{e}} \mathbf{p}^{2}-\frac{Z e^{2}}{\left(\rho^{2}+z^{2}\right)^{1 / 2}}+\frac{e^{2} B^{2}}{8 m_{e} c^{2}} \rho^{2} .
$$

The $z$ component of angular momentum $L_{z}$ is conserved, and so the kinetic-energy term in $H$ can be written as

$$
\frac{1}{2 m_{e}} \mathrm{p}^{2}=\frac{1}{2 m_{e}}\left(p_{\rho}^{2}+p_{z}^{2}\right)+\frac{L_{z}^{2}}{2 m_{e} \rho^{2}}
$$

and the azimuthal angle $\phi$ is an ignorable coordinate. In the low states of atoms, the diamagnetic term is usually negligible; however, in high- $n$ Rydberg states, this term is the source of interesting effects.

\section{B. Measurements of the absorption spectrum}

Garton and Tomkins were the first to observe diamagnetic effects in high- $n$ Rydberg states, and more precise measurements were later made by $\mathrm{Lu}$ et al. ${ }^{2}$ Some of their measurements are reproduced in Fig. 1. The lower part of the figure shows the absorption spectrum of the atom in the absence of a magnetic field. Each peak corresponds to a transition from the ground state into one of the high- $n$ states. Transitions into levels between $n=31$ and $n \sim 80$ are well resolved. At higher photon energies, the Rydberg levels are too closely spaced to be resolved, and we see a smooth average-absorption curve. There is no discontinuity at the ionization threshold: the average-absorption coefficient behaves continuously as the photon energy increases and the final states change from discrete bound states to continuous free-electron states.

When the magnetic field is turned on, the lower-energy levels $(n \sim 30-40)$ are split by the diamagnetic term. One can see that this splitting has an orderly pattern. For laboratory field strengths, this splitting is well described by first-order perturbation theory. ${ }^{3}$

In this experiment, higher levels $(n \sim 40-100)$ were not fully resolved, and at first glance the absorption spectrum appears to be very complicated. However, more recent, higher-resolution experiments carried out by the Massachusetts Institute of Technology (MIT) group have shown that many of the simple patterns that appear in the lower levels persist to some extent in these higher levels as well. ${ }^{4}$

Our present interest is in the absorption spectrum near the ionization threshold. There one sees that the absorption coefficient is an oscillatory function of energy. As the magnetic field strength $B$ increases, the "wavelength," or peak-to-peak energy spacing $\lambda_{E}$ of these oscillations, increases, and the amplitude of the oscillations also increases.

Higher-resolution measurements of near-threshold absorption in the hydrogen atom in a magnetic field have recently been carried out by the University of Bielefeld group, ${ }^{5}$ and one recent set of observations is reproduced in Fig. 2. At this higher resolution, the smooth oscillations seen in Fig. 1 are no longer visible, and the absorption as a function of energy seems to be quite chaotic.

In fact, however, this absorption signal is not at all chaotic: underlying it is a rather unexpected type of order. Let us denote the observed photon absorption plotted in Fig. 2 as $\mathcal{A}(E)$ (it is the measured photonabsorption rate in arbitrary units). The Fourier transform of this quantity, converting from energy to time as 


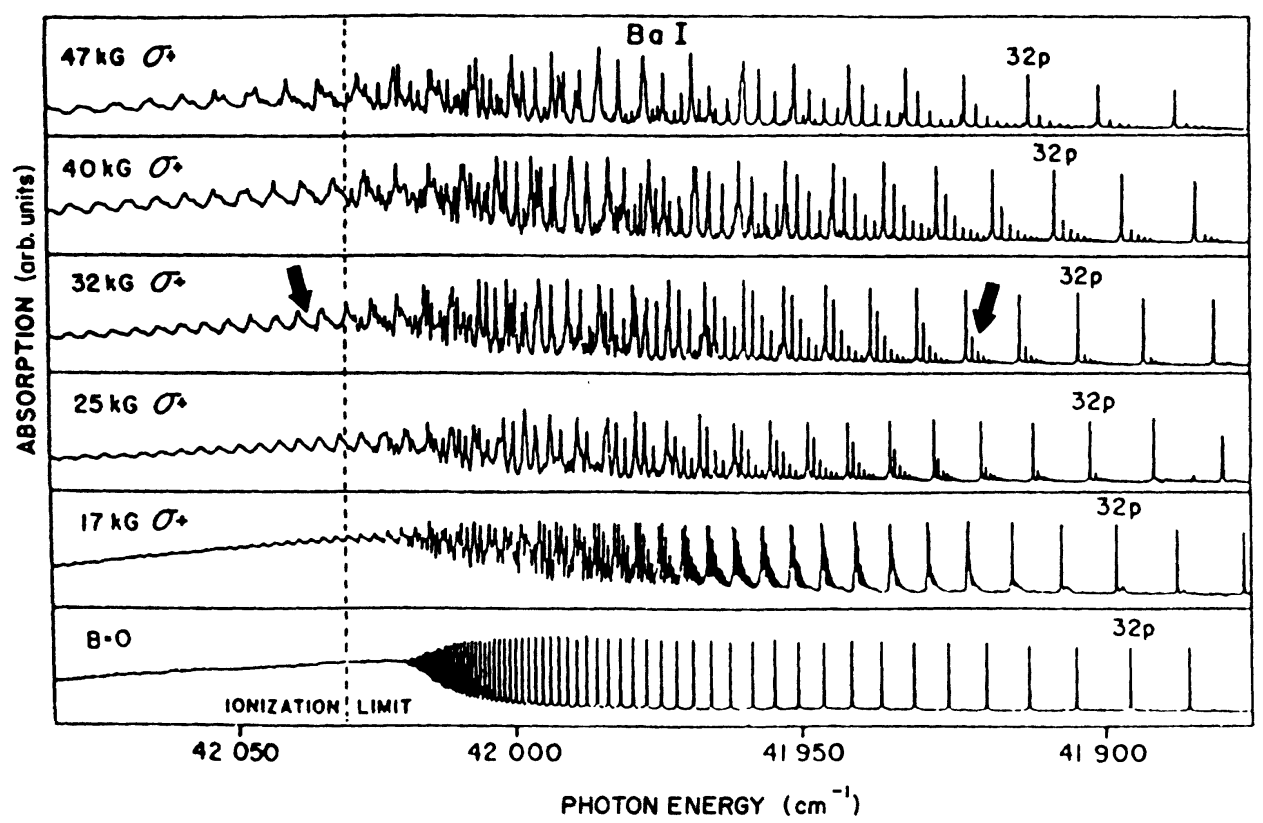

FIG. 1. Absorption spectrum of the barium atom close to the ionization threshold (from Ref. 2). Photon energy increases toward the left. Two distinct regions of the spectrum are visible. The arrow on the right points to a group of energy levels in the classically regular regime. Here the Rydberg levels are split in an orderly manner by the diamagnetic term. The arrow on the left points to oscillations that are visible in the spectrum in the classically chaotic regime. This paper provides a quantitative theory of such oscillations.

the independent variable, is denoted by $\tilde{\mathcal{A}}(T)$,

$$
\tilde{\mathcal{A}}(T)=\mathrm{const} \times \int_{E_{1}}^{E_{2}} \exp (-i E T / \hbar) \mathcal{A}(E) d E,
$$

where $E_{1}$ and $E_{2}$ are the lower and upper limits of the experimental measurements. This Fourier-transformed absorption signal, calculated in Ref. 5(b), is shown in Fig. 3 as the dotted line. Sharp peaks are visible at distinct points, $T_{n}$, on the time axis. The largest and lowestperiod peak corresponds to the oscillations seen by Gar-

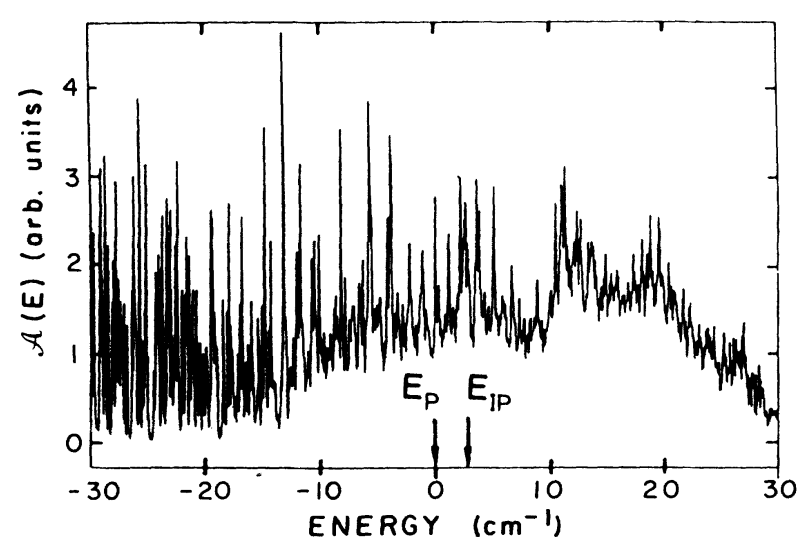

FIG. 2. Absorption spectrum of the hydrogen atom in a magnetic field $B=5.96 \mathrm{~T}$ [from Ref. 5(b)]. Photon energy increases to the right. The zero-field and actual ionization thresholds are indicated. The initial state was $2 p_{z}$, and the final states have $L_{z}=m \hbar=0$. At this higher resolution, the oscillations that are visible in Fig. 1 are not apparent to our eyes. They are still present however, as is shown by the Fourier transform. ton, Tomkins, and $\mathrm{Lu},{ }^{2}$ while the other peaks correspond to regular sinusoidal oscillations that have a shorter wavelength on the energy axis. Hence the seemingly chaotic absorption signal shown in Fig. 2 is actually a superposition of several distinct sinusoidal oscillations of wavelength $\lambda_{E}^{(n)}=2 \pi \hbar / T_{n}$.

The beginning of an interpretation of this phenomenon was provided by Edmonds. ${ }^{6}$ He showed that the oscillations observed by Garton and Tomkins were correlated with a periodic orbit of the electron moving under the

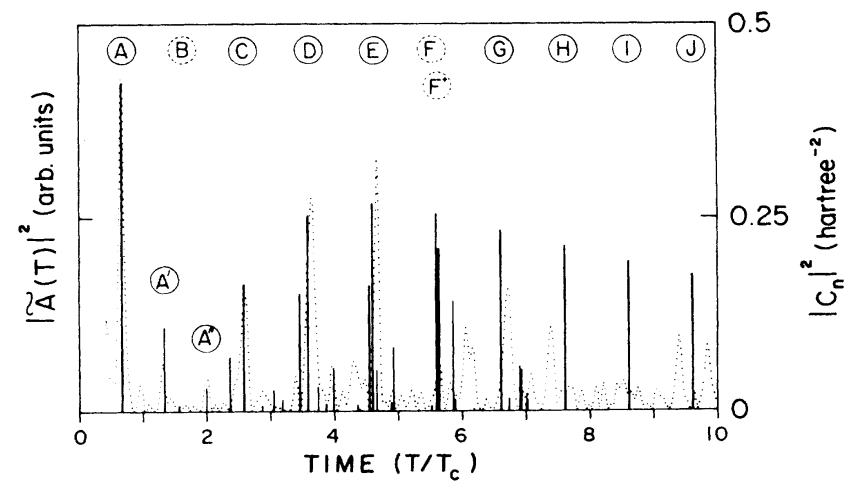

FIG. 3. The dotted line is the absolute square of the Fourier transform of the absorption spectrum [from Ref. 5(b)]. The unit of time is $T_{c}$, the cyclotron period of the electron. The sharp peaks show that $\mathcal{A}(E)$ is dominantly a superposition of sinusoidal oscillations. Each peak occurs at a time $T_{k}$ that corresponds to the time duration of a closed orbit that begins and ends at the nucleus. The vertical lines are our theoretical spectral-oscillation amplitudes $\left|C_{k}\right|^{2}$ computed at $E=0$ (Ref. 25). 
Hamiltonian (1.1). Motion along the $\rho$ axis is periodic, and the period of this motion turns out to be equal to $T_{1}$, the period of the largest peak in Fig. 3. Hence this classical periodic orbit is manifesting itself in the quantum spectrum as a sinusoidal oscillation in the average absorption as a function of energy, $\mathcal{A}(E)$.

Pursuant to this observation, classical trajectories were computed in Ref. 5(b), and it was found that most of the large peaks shown in Fig. 3 occur at times $T_{n}$ which are equal to periods of classical periodic orbits that begin and end close to the nucleus.

It so happens that the period $T_{1}$ is equal to $\frac{2}{3}$ of the cyclotron period for an electron in a magnetic field, so that the wavelength of the oscillations in $\mathcal{A}(E)$ is equal to $\frac{3}{2}$ of the spacing between Landau levels. Recognizing this, and perhaps thinking that the oscillations were caused by quasibound resonance states, Edmonds gave the name "quasi-Landau resonances" to this phenomenon.

More recently, Reinhardt ${ }^{7}$ attempted to make quantitative calculations of the absorption spectrum. It is known that the absorption spectrum $\mathcal{A}(E)$ can be related to a certain correlation function: let $\left|\psi_{i}\right\rangle$ be the initial state of the atom, let $D$ be the relevant component of the dipole operator, and let $\widehat{K}\left(t, t^{\prime}\right)$ be the evolution operator for the Hamiltonian (1.1). Then it can be shown that the observed absorption spectrum is proportional to the Fourier transform of the quantity

$$
k(t)=\left\langle D \psi_{i}|\hat{K}(t, 0)| D \psi_{i}\right\rangle
$$

In some of his calculations, Reinhardt replaced $\left|D \psi_{i}\right\rangle$ by a wave packet initially centered close to the nucleus, and he made some estimates of what the propagator would do to such a packet. In a semiclassical approximation, the packet will move along classical trajectories; some of those trajectories are periodic, and they return to the nucleus after time $T_{n}$. Therefore the matrix element $k(t)$ may have peaks at times $t=T_{n}$ corresponding to the periods of periodic orbits, and the Fourier transform $\mathcal{A}(E)$ would be expected to have oscillations of wavelength $\lambda_{E}=2 \pi \hbar / T_{n}$. This physical picture is helpful in many ways, ${ }^{7}$ but the attempt to obtain quantitative results from it was only partially successful.

The purpose of these papers ${ }^{8}$ is to provide a quantitative theory of the Garton-Tomkins-Edmonds phenomenon: we want to explain the existence of oscillations in the average-absorption spectrum of an atom in a magnetic field, and we want to show the relationship of such oscillations to classical periodic or closed orbits. In this first paper, the physical ideas are discussed in the simplest possible way. Since we know that the spectral oscillations are correlated with classical trajectories, we naturally give attention to the correspondence principle, and the general relationship between wave motion and particle motion. We shall see that classical trajectories in this system are generally chaotic. The classical limit of quantum mechanics for chaotic systems is not yet very well understood. Nevertheless, we shall show that a familiar correspondence principle, used in a less familiar way, leads to a quantitative theory of oscillatory spectra.

\section{RELATIONSHIP BETWEEN CLASSICAL TRAJECTORIES AND QUANTUM SPECTRA OR WAVE FUNCTIONS}

\author{
A. Semiclassical eigenfunctions \\ and the Einstein-Brillouin-Keller-Marcus scheme
}

The ideas underlying our analysis will be more clear if we briefly review the correspondence between wave functions and families of trajectories. ${ }^{9-11}$ As is well known, given an appropriate family of trajectories, we can associate with that family a vector field $\mathbf{p}(\mathbf{q})$ - the momentum as a function of position. If the trajectories are obtained from Hamiltonian equations of motion from properly formulated initial conditions, then associated with this field is a "characteristic function" $S(\mathrm{q})$, such that

$$
\begin{aligned}
& \mathbf{p}(\mathbf{q})=\nabla S(\mathbf{q}), \\
& S(\mathbf{q}):=\int^{\mathbf{q}} \mathbf{p}\left(\mathbf{q}^{\prime}\right) \cdot d \mathbf{q}^{\prime} .
\end{aligned}
$$

From the function $S(\mathbf{q})$, together with a density function $\rho(\mathbf{q})$ (which is also calculable from the family of trajectories and initial conditions), one can construct a wave function

$$
\psi(\mathbf{q})=|\rho(\mathbf{q})|^{1 / 2} \exp [i S(\mathbf{q}) / \hbar]
$$

and this wave function approximately satisfies the Schrödinger equation.

Usually, in practice, trajectories fold back over each other in such a way that more than one path passes through each point; then the vector field, characteristic function, and density function have several branches, and the wave function is a superposition of several terms,

$\psi(\mathbf{q})=\sum_{k}\left|\rho_{k}(\mathbf{q})\right|^{1 / 2} \exp i\left(S_{k}(\mathbf{q}) / \hbar-\mu_{k} \frac{\pi}{2}\right)$.

Here $\mu_{k}$ is the Maslov index, and it represents the relative phases of various terms in the superposition.

This approximation is commonly used to calculate energy eigenvalues and wave functions associated with regular trajectories. A regular quasiperiodic trajectory winds around in such a way that it by itself forms the required vector field $\mathbf{p}(\mathbf{q})$ [Fig. 4(a)]. This vector field supports a wave function of the form (2.2b) [Fig. 4(b)]. Careful examination of boundary conditions and phase relationships reveal that such a wave function can only be constructed if action integrals $\partial_{k}$ around distinct closed paths $c_{k}$ are quantized,

$$
\partial_{k}=\oint_{c_{k}} \mathbf{p}_{k}\left(\mathbf{q}^{\prime}\right) \cdot d \mathbf{q}^{\prime}=\left[n_{k}+\frac{\Delta \mu_{k}}{4}\right] 2 \pi \hbar .
$$

This procedure (the Einstein-Brillouin-Keller-Marcus, or EBKM, quantization scheme) therefore connects each individual eigenfunction to an individual quasiperiodic trajectory. 
Let us return now to the problem of oscillatory spectra, and the Garton-Tomkins-Edmonds phenomenon. From the early papers on this subject one might get the impression that appropriate applications of this quantization scheme would provide an explanation of the oscillations seen in the spectrum. Edmonds, ${ }^{6} \mathrm{Rau}^{12(\mathrm{a})}$ and Starace $^{12(b)}$ discussed the effect in terms of quantization of action integrals on a periodic orbit, and Al-Laithy et al. ${ }^{12(c)}$ considered the possibility of quantization of a second action variable representing motion across a periodic orbit. ${ }^{13}$

However, such an approach cannot be applied. Calculations show that in the relevant energy range, very few or perhaps none of the trajectories in the energy range of interest are quasiperiodic. Figure 5 shows a trajectory of the Hamiltonian (1.1) with a strong magnetic field and with energy just below the ionization threshold. This typical chaotic trajectory does not form any sort of smooth vector field $\mathbf{p}(\mathbf{q})$, and action integrals associated with this trajectory cannot be defined, so there is no way to build a wave function upon it using the EBKM scheme. Presently there exists no semiclassical theory connecting individual energy levels with individual irregular trajectories. As a consequence, it is not easy to say what properties individual eigenfunctions will have in the classical limit. ${ }^{14,15}$ A different approach is needed.

\section{B. Semiclassical Green's functions and the finite-resolution approximation}

An important idea comes from the nature of the experiments: ${ }^{5}$ in those measurements individual energy levels were not resolved; instead, an average absorption was measured to a band of states within the finite resolution of the laser. While presently no orderly pattern to individual eigenvalues has been seen, a simple pattern does exist for the spectrum at finite resolution-sinusoidal oscillations appear that are correlated with classical trajectories.

In these papers we shall obtain a semiclassical theory that describes the quantum spectrum at finite resolution. We consider measurements in which individual levels are
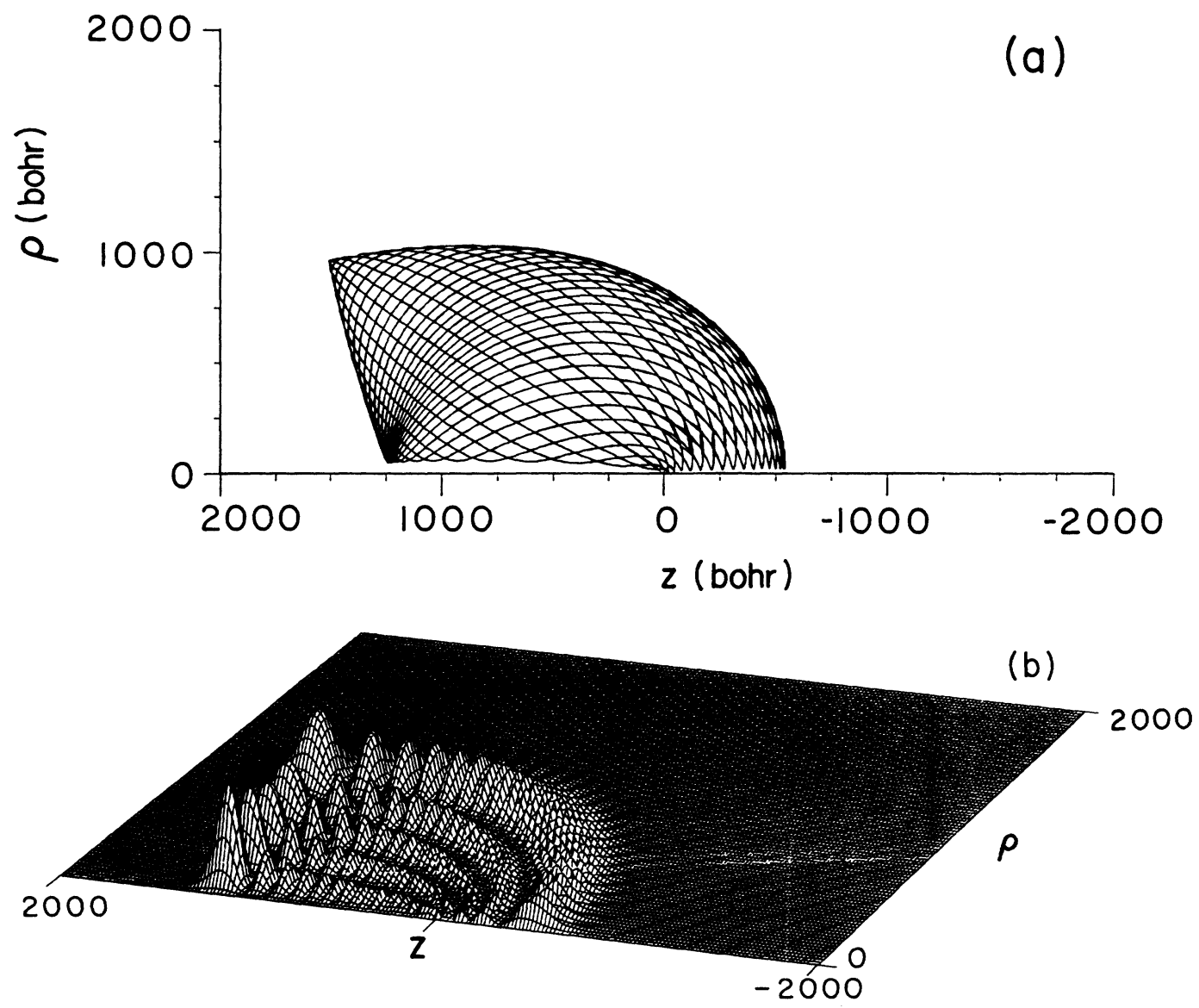

FIG. 4. (a) Regular trajectory of an atomic electron in Coulomb and diamagnetic fields (there is also a weak electric field pointing in the $-z$ direction, but it has little effect). The path followed by the electron is represented in cylindrical coordinates. This trajectory is one of the typical types that are associated with the regular part of the spectrum $(n \simeq 30, B \sim$ a few teslas). The momentum field has four branches. Associated with this momentum field is a semiclassical wave function given by Eq. (2.2b). (b) Quantum wave function correlated with the trajectory shown in Fig. 4(a). The wave function is large and oscillatory in the region of $(\rho, z)$ space occupied by the trajectory, and exponentially small in the rest of space. The number of oscillations in the wave function is correlated with classical action integrals. This wave function was calculated directly from quantum mechanics. The semiclassical approximation discussed in the text would give almost exactly the same result. 


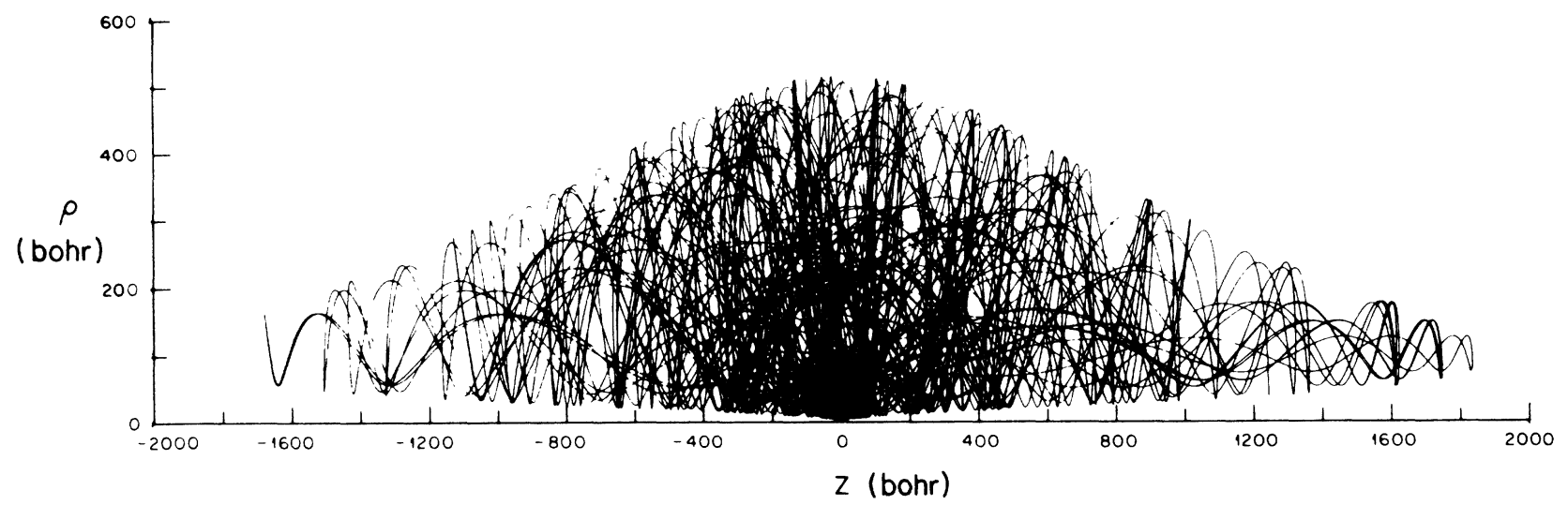

FIG. 5. Irregular trajectory of an atomic electron in Coulomb and diamagnetic fields. This is characteristic of the type of trajectory that occurs near the ionization threshold. There is no way to associate a smooth vector field with this trajectory, and therefore no way to build an eigenfunction from it.

not resolved, but in which fluctuations are seen in the average absorption as a function of energy.

The starting point of the theory is the Green's function $G_{E}^{+}\left(\mathbf{q} ; \mathbf{q}^{\prime}\right)$ - the wave produced at $\mathbf{q}$ by a steady source of outgoing waves of energy $E$ at $\mathbf{q}^{\prime}$ (Fig. 6). A semiclassical approximation to $G_{E}^{+}\left(\mathbf{q} ; \mathbf{q}^{\prime}\right)$ can be constructed from trajectories of energy $E$ that propagate radially outward from $q^{\prime}$. When the trajectories are propagated for a finite time, they form the required vector field $p(q)$, and the semiclassical approximation to the Green's function is

$$
\begin{aligned}
G_{E}^{+}\left(\mathbf{q} ; \mathbf{q}^{\prime}\right)=c \sum_{k} & \left|\rho_{k}\left(\mathbf{q} ; \mathbf{q}^{\prime}\right)\right|^{1 / 2} \\
& \times \exp \left(i S_{k}\left(\mathbf{q} ; \mathbf{q}^{\prime}\right) / \hbar-i \mu_{k} \frac{\pi}{2}\right) .
\end{aligned}
$$

Here we have explicitly noted the dependence of the classical functions $S$ and $\rho$ upon the selected initial point $\mathbf{q}^{\prime}$ as well as the final point $q$. The sum is again over all trajectories leading from $\mathbf{q}^{\prime}$ to $\mathbf{q}$, each of which corresponds to a branch of the trajectory field.

We need to know how this approximation to the Green's function changes as the energy changes. The classical functions $S_{k}\left(\mathbf{q} ; \mathbf{q}^{\prime}\right)$ and $\rho_{k}\left(\mathbf{q} ; \mathbf{q}^{\prime}\right)$ are evaluated on the ( $k$ th) trajectory of energy $E$ leading from $\mathbf{q}^{\prime}$ to $q$. As the energy changes slightly, this path shifts. A wellknown theorem in classical mechanics asserts that if the end points are held fixed, then the change of $S_{k}\left(q, q^{\prime}\right)$ with $E$ is given by

$$
\left(\frac{\partial S_{k}\left(\mathbf{q} ; \mathbf{q}^{\prime}\right)}{\partial E}\right)_{\mathbf{q}, \mathbf{q}^{\prime}}=T_{k},
$$

where $T_{k}$ is the time required for the particle to travel from $\mathbf{q}^{\prime}$ to $\mathbf{q}$ on the ( $k$ th) orbit of energy $E$. It follows that as $E$ changes, the real and imaginary parts of the $k$ th term in (2.4) oscillate with a wavelength in energy equal to $2 \pi \hbar / T_{k}$.

As it stands, Eq. (2.4) is not yet very helpful. For if the trajectories remain within a bounded region of space, then typically at any given energy an infinite number of trajectories can travel from $\mathbf{q}^{\prime}$ to $\mathbf{q}$. Normally the most direct path will arrive after a short time, and then there will be a sequence of other, ever-more-convoluted paths from initial to final points. According to Eq. (2.4), each such path would contribute a term to the Green's function. As $T_{k}$ increases, each term would become ever more sensitive to small changes in the energy, oscillating with a wavelength $\lambda_{E}=2 \pi \hbar / T_{k}$. Perhaps in the limit $\hbar \rightarrow 0$ an infinitely complicated interference structure could arise.

Suppose, however, that we only want to calculate $G_{E}^{+}\left(\mathbf{q} ; \mathbf{q}^{\prime}\right)$ to a finite resolution in energy. A finiteresolution Green's function would be an average of $G_{E}^{+}\left(q ; q^{\prime}\right)$ over a small energy range, and the averaging could involve a weighting function $g\left(E-E^{\prime}\right)$,

$$
\bar{G}_{E}\left(\mathbf{q} ; \mathbf{q}^{\prime}\right)=\int G_{E^{\prime}}^{+}\left(\mathbf{q} ; \mathbf{q}^{\prime}\right) g\left(E-E^{\prime}\right) d E^{\prime} \text {. }
$$

Since long-period orbits produce terms in (2.4) that would be averaged to zero by such a process, it follows that they could be neglected in the calculation of the finiteresolution Green's function.

The above argument helps to justify our fundamental proposition.

A semiclassical approximation to a finite-resolution Green's function $\bar{G}_{E}\left(\mathbf{q} ; \mathbf{q}^{\prime}\right)$ can be calculated by including in the sum (2.4) only those terms arising from trajectories which travel from $\mathbf{q}^{\prime}$ to $\mathbf{q}$ in a finite time $T_{k} \leq T_{\max }$. The resolution $\Delta E$ associated with this function $\overline{\bar{G}}_{E}$ is related to $T_{\max }$ by $\Delta E T_{\max } \simeq 2 \pi \hbar$.

\section{Green's function and the spectrum}

\section{Density of states}

A simple and well-known formula permits us to calculate the density of states from the Green's function,

$$
\rho(E)=-\frac{1}{\pi} \int \operatorname{Im}\left[G_{E}^{+}(\mathbf{q}, \mathbf{q})\right] d \mathbf{q} .
$$



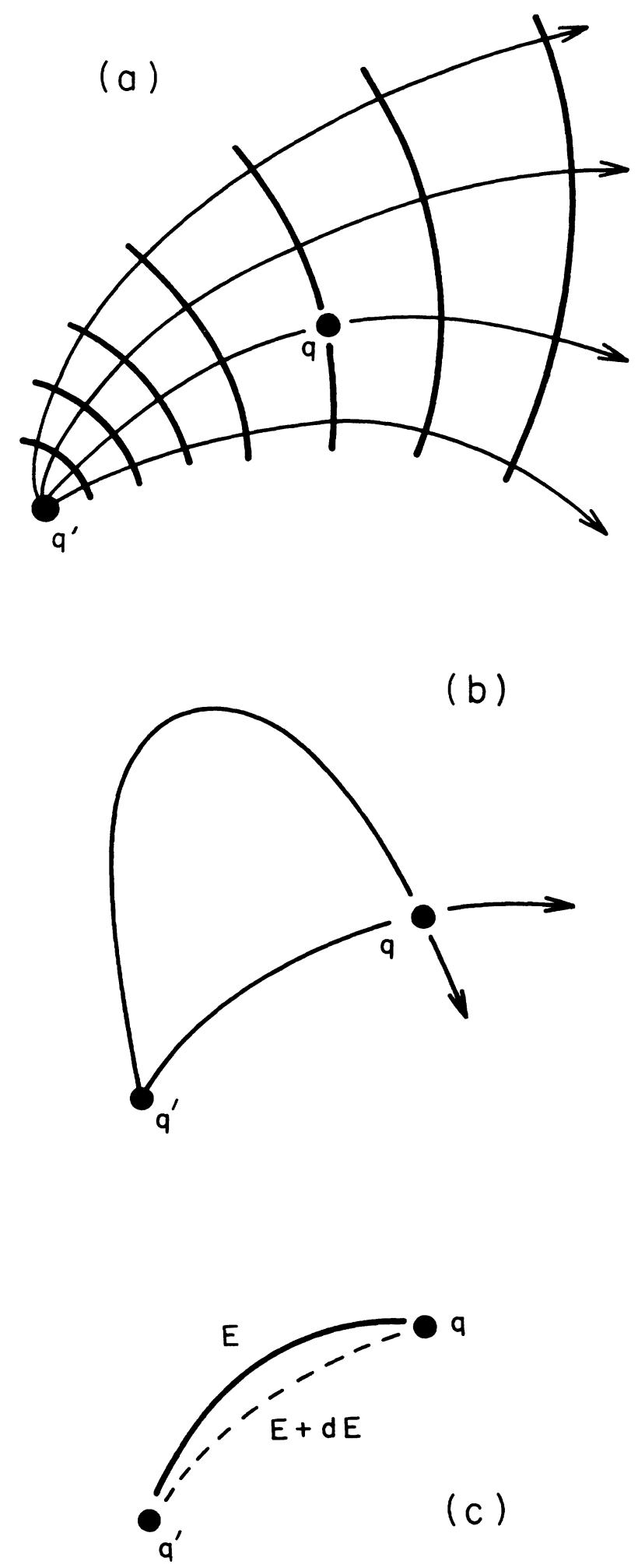

FIG. 6. (a) Schematic representation of the Green's function as the wave at $q$ raising from a source at $q^{\prime}$. It can be calculated from the properties of the trajectory of energy $E$ from $\mathbf{q}^{\prime}$ to $\mathbf{q}$. (b) If two distinct trajectories of energy $E$ propagate from $q^{\prime}$ to $q$, then $G\left(q, q^{\prime} ; E\right)$ is a sum. (c) As the energy changes the trajectory from $q^{\prime}$ to $q$ changes slightly, and the classical action changes according to $\partial S / \partial E=T$.
To obtain a semiclassical approximation to the finiteresolution density of states, we could use Eq. (2.4) together with Eq. (2.7). Actually, the function $G_{E}^{+}(\mathbf{q} ; \mathbf{q})$ consists of two parts: (i) a singular term arising from the fact that the source point $q^{\prime}$ is the same as the field point $q$ and (ii) a set of semiclassical terms of the form (2.4), each of which represents waves that propagate out from the source point $\mathbf{q}^{\prime}$, travel around in space for a while, and later return to pass through the field point $q$, which is at the same spot as the source point. When the integral (2.7) is evaluated, it is found that the singular term in $G_{E}^{+}(q ; q)$ gives the familiar "phase-space-volume" formula for the density of states,

$$
\rho_{0}(E)=(2 \pi \hbar)^{-n} d \Omega(E) / d E,
$$

where $\Omega(E)$ is equal to the volume of phase space surrounded by the energy surface $H(\mathrm{p} ; \mathrm{q})=E$.

The semiclassical terms $(2.4)$ in $G_{E}(q ; q)$, representing waves returning to the source point, give oscillatory corrections to the density of states. When the integral (2.7) is evaluated using the stationary-phase method, it turns out that only periodic orbits contribute significantly, and each such orbit contributes a term of the form

$$
\rho_{k}(E)=a_{k}(E) \sin \left[S_{k}(E) / \hbar+\eta_{k}\right]
$$

From Eq. (2.5), it follows that this term oscillates as a function of energy with a wavelength equal to $2 \pi \hbar / T_{k}(E)$, where $T_{k}(E)$ is the period of the orbit.

The full semiclassical approximation to the density of states is then given by the sum of the slowly varying "phase-space" term plus the oscillatory "periodic-orbit" terms,

$$
\rho(E)=\rho_{0}(E)+\sum_{k} \rho_{k}(E)
$$

It should be emphasized that in this method it is not claimed that any relationship exists between individual orbits and individual quantum states. It is rather that when (2.4) is used in (2.7), then each term in (2.4) contributes a sinusoidal oscillation to $\rho(E)$. Restricting the sum (2.10) to a finite set of orbits, we then obtain a semiclassical approximation to the finite-resolution density of states. If the sum (2.10) "converges," then at certain distinct energies, oscillations from many terms in (2.4) interfere constructively, thus leading to sharp peaks at the semiclassical eigenvalues.

Gutzwiller ${ }^{16}$ and Balian and Bloch ${ }^{17}$ were the first to develop a theory of this type, and the approach was later applied by Berry and Tabor to regular systems, ${ }^{18}$ and by Berry to certain chaotic systems. ${ }^{19}$ Generally those authors were seeking individual eigenvalues for chaotic systems, and they found that summations over very large numbers of periodic orbits would be required. Therefore it is not yet known whether this really provides a practical scheme for computing individual eigenvalues. (Questions about convergence, and about the limits $\hbar \rightarrow 0$ and $T_{k} \rightarrow \infty$ also arise. ${ }^{20}$ ) 


\section{Oscillator strengths}

Spectroscopic measurements at finite resolution do not give the density of states. Instead, they give an average oscillator-strength density. For a well-resolved discrete transition between initial state $\psi_{i}$ and final state $\psi_{f}$, the oscillator strength is defined as

$$
f_{f i}=\frac{2 m_{e}\left(E_{f}-E_{i}\right)}{\hbar^{2}}\left|\left\langle\psi_{f}|D| \psi_{i}\right\rangle\right|^{2},
$$

where $D$ is the component of the dipole operator in the direction of polarization of the exciting electromagnetic field. The rate of absorption of photons, or the rate of production of atoms in excited states, is directly proportional to this oscillator strength.

When transitions occur from a single initial state to a group of unresolved final states, then the total transition rate is proportional to an average oscillator-strength density, defined approximately as

$$
\overline{D f}(E)=\int f_{f i} \rho\left(E_{f}\right) g\left(E-E_{f}\right) d E_{f},
$$

where again $g\left(E-E_{f}\right)$ is a convolution function with width in energy comparable to the resolution of the laser.

Just as for the density of states, a simple formula relates the oscillator-strength density to the Green's function,

$$
\begin{aligned}
D f\left(E_{f}\right) & \equiv f_{f i} \rho\left(E_{f}\right) \\
& =-\frac{2 m_{e}\left(E_{f}-E_{i}\right)}{\pi \hbar^{2}} \operatorname{Im}\left\langle D \psi_{i}\left|G_{E_{f}}^{+}\right| D \psi_{\imath}\right\rangle .
\end{aligned}
$$

The same formula relates the average oscillator-strength density to the finite-resolution Green's function

$\overline{D f}(E)=-\frac{2 m_{e}\left(E-E_{i}\right)}{\pi \hbar^{2}} \operatorname{Im}\left\langle D \psi_{i}\left|\bar{G}_{E}\right| D \psi_{i}\right\rangle$.

We shall show that this formula, together with the semiclassical approximation (2.4), using orbits of duration up to some maximum $T$, provides a practical method for computing the spectrum.

Several aspects of this problem make it simpler than the problems studied in Refs. 16-19. (1) We are not trying to calculate individual eigenvalues or the oscillatorstrength to individual final states. We only want the absorption spectrum to a resolution comparable to (or we hope a little better than) that obtained in the experiments. (2) The initial state is localized. Therefore we only need $\bar{G}_{E}\left(\mathbf{q} ; \mathbf{q}^{\prime}\right)$ in a limited domain of space. (3) Close to the nucleus, a semiclassical approximation cannot be applied. However, in this region the diamagnetic field can be neglected, so the Hamiltonian is spherically symmetric. (4) It follows that in this region, the angular dependence of $\left|D \psi_{i}\right\rangle$ and of $\bar{G}_{E}\left|D \psi_{i}\right\rangle$ is simple. Their expansions in partial waves typically contain between one and three terms. For this reason we actually use a semiclassical approximation to $\bar{G}_{E}\left|D \psi_{i}\right\rangle$ rather than to $\bar{G}_{E}$ itself. (5) We only make a calculation of the absorption spectrum in a narrow range of energies, close to the ion- ization threshold.

Each of these aspects of the problem leads to useful simplifications. Finally, it is important to note that because the semiclassical approximation and an associated stationary-phase approximation are not appropriate in the vicinity of the nucleus, we find that not only periodic orbits, but also closed orbits, must be included.

\section{THEORY OF OSCILLATORY SPECTRA}

\section{A. Central idea}

Equations (2.13b) and (2.4) lead to a simple physical picture as well as a quantitative theory explaining how oscillations in the absorption spectrum are correlated with closed classical orbits. (See Fig. 1 of accompanying paper. ${ }^{8}$ ) When the atom absorbs a photon, the electron goes into a near-zero-energy outgoing Coulomb wave. This wave propagates away from the nucleus to large distances. For $r \gtrsim 50 a_{0}$ the outgoing wave fronts propagate according to semiclassical mechanics, and they are correlated with outgoing classical trajectories. Eventually the trajectories and wave fronts are turned back by the magnetic field; some of the orbits return to the nucleus, and the associated waves (now incoming) interfere with the outgoing waves to produce the observed oscillations.

Specifically, Eqs. (2.4) and (2.5) tell us that as the energy changes, the imaginary part of each term in (2.4) oscillates with a wavelength on the energy axis of $\lambda_{E}=2 \pi \hbar / T_{k}$. Here $T_{k}$ is the duration of a closed orbit which begins and ends in the vicinity of the nucleus, such that the corresponding waves overlap with the initial state.

A useful general concept can be abstracted from these formulas. Even in a classically chaotic system, order exists on a finite time scale. For any finite time, the solutions $\mathrm{q}\left(t, \mathrm{q}^{0}\right)$ to smooth classical equations of motion (2.1) are smooth functions of the initial conditions. As time increases, these functions may become so stretched and convoluted that for all practical purposes the relationship between the initial point and the final point is lost. However, if trajectories are examined on a limited time scale, then the smooth relationship will be clear (Fig. 7).

One of the consequences of this finite-time order in classical mechanics is the orderly sinusoidal oscillations that occur in finite-resolution quantum spectrum. Surrounding each closed orbit is a family of orbits traveling in step with that orbit, but gradually moving away from it. Associated with each such family of orbits is a propagating wave, which is as orderly as the family of orbits. ${ }^{21}$ Thus, though we might not be able to construct a wave function from a single chaotic orbit (such as the one shown in Fig. 5), or from a family propagated for a long time [as in Fig. 7(b)], there is a wave associated with a family propagating for a short time. Interference of these waves with the outgoing wave and with each other produces oscillations which are visible in the spectrum if it is measured to finite resolution.

\section{B. Quantitative formulas}

To obtain a quantitative description of the observed spectral oscillations, several rather detailed technical 

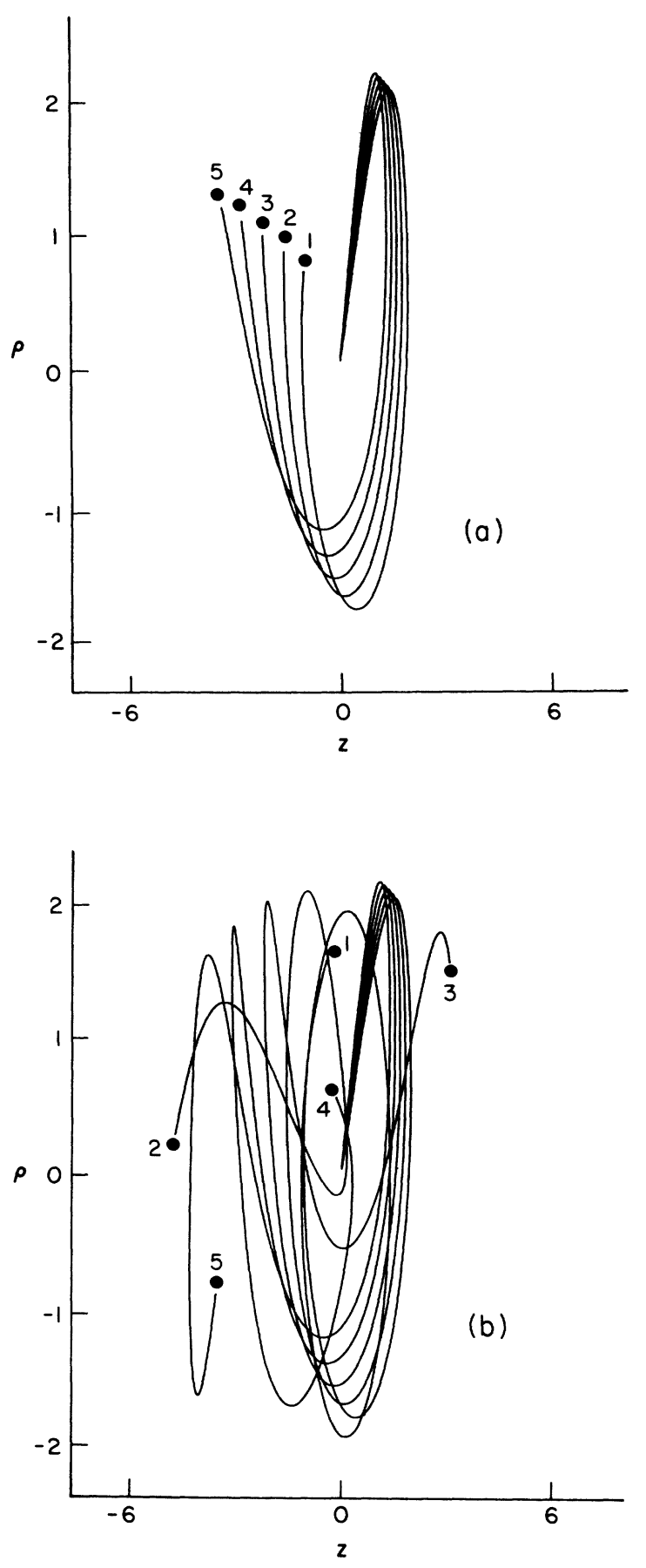

FIG. 7. Short-term order, long-term chaos. For the Hamiltonian (1.1), five trajectories were launched from the nucleus starting with identical energies and slightly different directions. (a) When they are followed for a short time (to $T=1.5 T_{c}$, where $T_{c}$ is the cyclotron period) the smooth relationship between initial direction and final position is evident. (b) The same trajectories evolve for a longer time, to $T=3.0 T_{c}$. In principle, a smooth relationship between initial direction and final position still exists, but the relationship is now so convoluted that to our eyes, chaos has appeared. A wave function of the form (2.2b) can be correlated with an orderly family. However, Eq. (2.2b) might not apply after the family has broken up. problems have to be solved.

(1) Of course, we need the initial state; in this case it is a hydrogenic $2 p$ state. Combining it with the appropriate component of the dipole operator, one obtains for $\left|D \psi_{i}\right\rangle$ a combination of partial waves times Clebsch-Gordan coefficients. The result is written in the form

$$
D \psi_{i}=r R_{n l}(r) \sum_{l, m} b_{l m}^{i} Y_{l m}(\theta, \phi),
$$

where $b_{l m}^{i}$ are coefficients derivable from the polarization of incident radiation.

(2) The outgoing zero-energy Coulomb wave is $G_{E=0}^{+}\left|D \psi_{i}\right\rangle$. Close to the nucleus (i.e., $r \lesssim 50 a_{0}$ ) the diamagnetic field is neglected, and $G_{E=0}^{+}$is itself expanded in partial waves. The familiar radial Green's functions

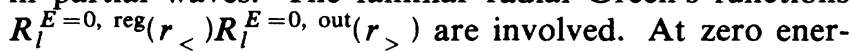
gy, the radial Coulomb wave functions are Bessel functions,

$$
\begin{aligned}
& R_{l}^{E=0, \operatorname{reg}}(r)=J_{2 l+1}\left((8 r)^{1 / 2}\right) / r^{1 / 2}, \\
& R_{l}^{E=0, \text { out }}(r)=H_{2 l+1}\left((8 r)^{1 / 2}\right) / r^{1 / 2} .
\end{aligned}
$$

The action of $G_{E=0}^{+}$upon $\left|D \psi_{i}\right\rangle$ therefore involves an

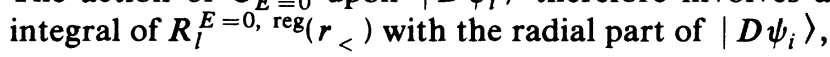

$I\left(n, l, l^{\prime}\right) \equiv \int_{0}^{\infty} d r r^{3} R_{n l}(r) J_{2 l^{\prime}+1}\left((8 r)^{1 / 2}\right) / r^{1 / 2}$.

This integral can be evaluated analytically.

(3) At distances around $50 a_{0}$, the asymptotic (large- $r$ ) form for $G_{E}^{+}|D \psi\rangle$ has to be connected to a semiclassical form. This connection is not unique, and we choose the simplest way to make it. On a boundary sphere of radius $50 a_{0}, G_{E}^{+}|D \psi\rangle$ is evaluated using Eqs. (3.2) together with the asymptotic form for the Hankel function. We then regard this quantity, $G_{E}^{+}\left|D \psi_{i}\right\rangle$, as a cylindrical outgoing wave. Semiclassical propagation of such a wave involves the initial conditions

$$
\begin{aligned}
& r=r_{b}, \\
& \theta=\theta_{i} \quad(\text { points on a dense grid), } \\
& \phi=\text { ignorable }, \\
& p_{\phi}=m \hbar, \\
& p_{\theta}=0, \\
& p_{r}=\left[2 m_{e}\left[E+\frac{1}{r}-\frac{p_{\phi}^{2}}{2 m_{e} r^{2}}\right]\right]^{1 / 2} .
\end{aligned}
$$

The amplitude of the wave on the initial surface is obtained from the $\theta$ dependence of $G_{E}^{+}\left|D \psi_{i}\right\rangle$ (partial-wave expansion).

(4) At larger distances, the waves propagate according to semiclassical mechanics, following classical trajectories. The azimuthal angle $\phi$ is an ignorable coordinate, so the calculation of trajectories, actions $S$, and classical densities $\rho$ involves the integration of only two coordinates, $r(t)$ and $\theta(t)$. For the relevant fixed value of $m=L_{z} / \hbar$, orbits are integrated numerically, and the computer searches for those orbits that return to the initial sphere in a time less than $T_{\max }$. Those orbits are la- 
beled by $m$ and an index $k_{m}$ (we refer to the $k_{m}$ th closed orbit in the $m$ th subspace). For each such orbit, the computer returns the following.

oThe initial and final values of the polar angle $\theta_{i}^{m k_{m}} \theta_{f}^{m k_{m}}$.

-The (two-dimensional) action on the path from the initial point to the final point on the sphere, $S_{2}^{m k_{m}}=\int\left(p_{r} \dot{r}+p_{\theta} \dot{\theta}\right) d t$.

OThe (two-dimensional) classical amplitude at the final point on the orbit,

$$
A_{2}^{m k_{m}}=\left(\frac{J_{2}(0)}{J_{2}(t)}\right)^{1 / 2}=\rho_{m k_{m}}^{1 / 2}
$$

where the Jacobian is

$$
J_{2}(t)=\left|\frac{\partial(r, \theta)}{\partial\left(t, \theta_{i}\right)}\right|
$$

( $A_{2}^{m k_{m}}$ is by definition positive.) The Maslov index $\mu_{m k_{m}}$ for each orbit was obtained by inspection of a graph of the orbit.

(5) The returning waves propagate inward from the sphere at $50 a_{0}$ toward the nucleus, where they overlap with $\left\langle D \psi_{i}\right|$. In this region the semiclassical approximation is no longer reliable. We make the approximation that the returning waves are cylindrically modified zeroenergy Coulomb scattering waves. For $r \lesssim 50 a_{0}$ the returning waves (which originally went out from the sphere, traveled to distances of $1000 a_{0}$ or more, then turned around and came back to the original sphere) look very much like wave functions for an electron that comes in with zero energy from infinite distance at polar angle $\theta_{f}$ interacting only with the Coulomb field.
This cylindrically modified zero-energy Coulombscattering wave is denoted $\psi_{c}^{m, \theta_{f}}(r, \theta, t)$. We need its asymptotic form and its partial-wave expansion. Derivation of the required formulas is straightforward but long.

(6) Finally, the semiclassical returning wave on the sphere $r \approx 50 a_{0}$ is set equal to a constant times $\psi_{c}^{m, \theta_{f}}(r, \theta, t)$. The constant is evaluated by comparing the value of the semiclassical returning wave at $r=50, \theta=\theta_{f}$ with that of $\psi_{c}^{m, \theta_{f}}\left(r, \theta=\theta_{f}, \phi\right)$. Then the partial-wave expansion of $\psi_{c}^{m, \theta_{f}}$ allows us to continue the returning wave in toward the nucleus. Only the first few terms in this partial-wave expansion contribute to the overlap with $\left\langle D \psi_{i}\right|$. The Bessel functions and integrals $I\left(n, l, l^{\prime}\right)$ enter the formulas a second time.

This argument leads to a simple formula for the average oscillator-strength density $\overrightarrow{D f}(E)$. We consider the case that the initial state and light polarization are chosen such that the states excited by the laser have a unique value of $L_{z}=m \hbar$. Then the formula for the average oscillator-strength density near $E=0$ is

$\overline{D f}(E)=D f_{0}(E)+\sum_{k_{m}} C_{m k_{m}} \sin \left(T_{m k_{m}} E+\Delta_{m k_{m}}\right)$.

$D f_{0}$, the smooth background term, is equal to the oscillator-strength density that would be obtained in the absence of a magnetic field,

$$
D f_{0}(E=0)=-\frac{4 m_{e} E_{i}}{\hbar^{2}} \sum_{l^{\prime}}\left|b_{l^{\prime} m}^{i} I\left(n, l, l^{\prime}\right)\right|^{2} .
$$

Each oscillatory term arises from a closed orbit, labeled $k_{m}$. The amplitude and phase constants, $C_{m k_{m}}$ and $\Delta_{m k_{m}}$, are calculated from the formula

$$
\begin{aligned}
C_{m k_{m}} \exp \left(i \Delta_{m k_{m}}\right)= & \left(-E_{i}\right) 2^{19 / 4} \pi^{3 / 2} r_{b}^{-1 / 4}\left(\sin \theta_{i}^{m k_{m}} \sin \theta_{f}^{m k_{m}}\right)^{1 / 2}\left|\rho_{m k_{m}}\right|^{1 / 2} \\
& \times \operatorname{expi}\left(S^{m k_{m}}-\frac{\pi}{2} \mu^{m k_{m}}+2\left(8 r_{b}\right)^{1 / 2}-\frac{3 \pi}{4}\right) \mathcal{Y}\left(\theta_{i}^{m k_{m}}\right) \mathcal{Y}^{*}\left(\theta_{f}^{m k_{m}}\right),
\end{aligned}
$$

where

$$
\mathcal{Y}(\theta)=\sum_{l^{\prime}}(-1)^{l^{\prime}} b_{l^{\prime} m}^{i} I\left(n, l, l^{\prime}\right) Y_{l^{\prime} m}(\theta, 0) .
$$

$C_{m k_{m}}$ and $\Delta_{m k_{m}}$ are obtained by evaluating the amplitude and phase of the right-hand side of Eq. (3.7a).

Finally, $T_{m k_{m}}$ is the time required for the $k_{m}$-th orbit to travel out from and return to the boundary sphere. Therefore Eq. (3.5) is a combination of a term that is slowly varying with energy (constant, at this level of approximation) together with a set of terms having wavelength in energy $\lambda_{E}=2 \pi \hbar / T_{m k_{m}}$. The amplitudes and phase constants of each oscillatory term are all calculable in a straightforward way from the properties of the corresponding closed orbit, together with formulas for the dipole integrals $I\left(n, l, l^{\prime}\right)$.

The derivation of Eqs. (3.5)-(3.7) is presented in the accompanying paper. ${ }^{8}$ We hope the meaning of these equations is clear from the preceding discussion. Notation is summarized in Table $I$.

\section{CALCULATION OF SPECTRAL OSCILLATIONS}

We now use Eqs. (3.1)-(3.7) to calculate near-threshold spectral oscillations for a hydrogen atom in a magnetic field. We consider a field $B=5.96 \mathrm{~T}$, and we examine three particular sets of transitions

$$
\begin{aligned}
& 2 p_{m=0} \rightarrow m=0, \quad E \simeq 0, \\
& 2 p_{m= \pm 1} \rightarrow m=0, \quad E \simeq 0, \\
& 2 s \rightarrow m=0, \quad E \simeq 0 .
\end{aligned}
$$

For the first of these, experimental measurements are available, and we will compare our calculations to those 
TABLE I. Notation used in Eqs. (3.5)-(3.7).

\begin{tabular}{|c|c|}
\hline$D f(E)$ & $\begin{array}{l}\text { Average oscillator-strength density, proportional } \\
\text { to the average rate of absorption of photons }\end{array}$ \\
\hline$D f_{0}(E)$ & $\begin{array}{l}\text { Smooth background, equal to the average } \\
\text { oscillator-strength density in the absence } \\
\text { of external fields }\end{array}$ \\
\hline$m k_{m}$ & $\begin{array}{l}\text { Label for the } k_{m} \text {-th closed orbit in the subspace } \\
\qquad L_{z}=m \hbar\end{array}$ \\
\hline$C_{m k_{m}}$ & Spectral-oscillation amplitude \\
\hline$T_{m k_{m}}$ & $\begin{array}{l}\text { Time for the }\left(m k_{m}\right) \text { th closed orbit to travel } \\
\text { out from and back to the boundary sphere }\end{array}$ \\
\hline$\Delta_{m k_{m}}$ & Absolute phase of the spectral oscillations \\
\hline$I\left(n, l, l^{\prime}\right)$ & $\begin{array}{l}\text { Radial dipole integrals involving the initial } \\
\text { state } R_{n l}(r) \text { and the regular zero-energy } \\
\text { Coulomb wave function } J_{2 l^{\prime}+1}\left[(8 r)^{1 / 2}\right] / r^{1 / 2}\end{array}$ \\
\hline$b_{l m}^{l}$ & $\begin{array}{l}\text { Coefficients for the partial-wave expansion } \\
\text { of }\left|D \psi_{i}\right\rangle\end{array}$ \\
\hline$r_{b}$ & $\begin{array}{l}\text { Radius of the boundary sphere; outside this radius } \\
\text { the waves propagate semiclassically }\end{array}$ \\
\hline \multicolumn{2}{|c|}{ For the $\left(m k_{m}\right)$ the closed trajectory } \\
\hline$\theta_{\imath}^{m k}{ }_{m}^{m} \theta_{f}^{m k}$ & $\begin{array}{l}\text { Initial and final polar angles, where it passes } \\
\text { through } r_{b}\end{array}$ \\
\hline$S^{m k}$ & Classical action \\
\hline$\left|\rho_{m k_{m}}\right|^{1 / 2}=A_{2}^{m k}$ & Classical density, positive by definition \\
\hline$\mu_{m k_{m}}$ & Maslov index \\
\hline
\end{tabular}

results. For the last two cases, measurements have not yet been made.

We shall describe our calculations on the first case in detail. The other cases are done by the same method, and only minor modifications are required.

\section{A. Transition $2 p_{m=0} \rightarrow m=0, E \simeq 0$ \\ 1. Background spectrum}

For the initial state $2 p_{z}$, the wave function is

$$
\psi_{i}=R_{21} Y_{10}
$$

and its energy (in hartree units) is

$$
E_{i}=-\frac{1}{8}
$$

For transitions to final states with the same magnetic quantum number $m_{f}=m_{i}=0$, the light must be polarized with electric field along the $z$ axis, so

$$
D=z=r \cos \theta \text {. }
$$

We combine Eqs. (4.1) and (4.3), using familiar properties of angular momentum eigenfunctions. ${ }^{22}$ Then, using Eq.
(4.2) of Ref. 8(a), $D \psi_{i}$ can be written as

$$
D \psi_{i}=r R_{21}\left[\left(\frac{4}{15}\right)^{1 / 2} Y_{20}+\left(\frac{1}{3}\right)^{1 / 2} Y_{00}\right] .
$$

Expressing this in the notation of Eq. (3.2), we find

$$
\begin{aligned}
& D \psi_{i}=\left(\sum_{l^{\prime} m} b_{l^{\prime} m}^{i} Y_{l^{\prime} m}\right) r R_{n l}(r), \\
& b_{20}^{i}=\left(\frac{4}{15}\right)^{1 / 2}, \quad b_{00}^{i}=\left(\frac{1}{3}\right)^{1 / 2},
\end{aligned}
$$

and all other $b_{l^{\prime} m}^{i}$ are zero.

The smooth background absorption $D f_{0}(E)$ is the absorption that arises in the absence of a magnetic field. The relevant dipole matrix elements are defined in Eq. (3.3). From Eq. (3.6), we find

$D f_{0}(E=0)=\frac{1}{2}\left\{\frac{4}{15}[I(2,1,2)]^{2}+\frac{1}{3}[I(2,1,0)]^{2}\right\}$.

These integrals are tabulated in paper II; the two required values are

$$
I(2,1,2)=5.4142, \quad I(2,1,0)=1.3535 .
$$

Therefore the background absorption is

$$
D f_{0}(E=0)=4.2138(\text { hartrees })^{-1} \text {. }
$$


$D f_{0}(E)$ is a slowly varying function, and we shall approximate it by the preceding constant value over the entire energy range of our calculations. Superimposed on this background are oscillations correlated with closed orbits in the system.

\section{Trajectories and closed orbits in the subspace $m=0$}

To compute the oscillations in the spectrum we have to study the classical trajectories of this system. Because only $m=0$ spherical harmonics appear in the expansion of $D \psi_{i}$ in Eq. (4.4), it is enough to restrict ourselves to the $m=0$ subspace.

The natural time unit in this problem is the cyclotron period

$$
T_{c}=\frac{2 \pi m_{e} c}{e B}=6.0 \times 10^{-12} \mathrm{~s}
$$

The available experimental spectrum corresponds to trajectories which return to the nucleus within a time $T \lesssim 10 T_{c}$. We shall find those closed orbits.

For $m=0$, closed orbits may be said to begin and end exactly at the nucleus. More conveniently, however, they may be defined as those orbits that leave the sphere $r_{b}$ and later reenter the sphere $r_{b}$ with $p_{\theta}$ exactly equal to zero. (Note that they do not necessarily return in the direction from which they left.) Since $m$ is always equal to zero in the present calculation, the closed orbits can be specified by a single label $k$ instead of the double label $\left(m k_{m}\right)$ used elsewhere.

We choose initial coordinates $r=r_{b}, \theta=\theta_{i}$, and initial momenta according to Eq. (3.4), with $p_{\phi}=0$ and $E=0 . \theta_{i}$ is the initial polar angle from the $z$ axis. In principle, all the $\theta_{i}$ from $0^{\circ}$ to $180^{\circ}$ should be examined. But because the Hamiltonian has a symmetry in $z$, the trajectory going out at angle $\theta_{0}$ can be obtained by a reflection about the $\rho=0$ axis from the trajectory going out at angle $180^{\circ}-\theta_{0}$. Therefore we only need to launch trajectories with $0^{\circ} \leq \theta_{0} \leq 90^{\circ}$.

Even with these restrictions and simplifications, the number of such closed orbits may be large. Nevertheless, we can find the important closed orbits of the system. Each closed orbit (which returns exactly to the nucleus) is surrounded by neighbors which return to the vicinity of the nucleus. The important closed orbits are those with large classical amplitude factors $A_{2}^{k}$. These amplitudes are inversely related to the rate of divergence of neighbors from the central closed orbit. In other words, the important closed orbits are the ones from which the neighbors diverge relatively slowly. We find these as follows. Trajectories are launched from the initial circle $r=r_{b}$ in all directions between $0^{\circ}$ and $90^{\circ}$ with initial angle spacing $\Delta \theta_{0}$ for neighboring trajectories; then the trajectories are computed and the coordinates $(r, \theta)$ and momenta $\left(p_{r}, p_{\theta}\right)$ are monitored. A trajectory returning to the vicinity of the nucleus crosses the circle $r=r_{b}$. If $\Delta \theta_{0}$ is small, we usually find families of neighboring trajectories that cross the circle. The number of such trajectories in a family is a measure of the divergence of this family of trajectories. The greater the number, the more stable this family. We call this number the "importance number" for this family, and denote it by $N_{\text {imp }} \cdot{ }^{23}$ In each family of trajectories, there will be a central orbit which satisfies $p_{\theta}=0$ when it reenters the sphere $r=r_{b}$. This closed orbit can easily be found by an iteration procedure once two trajectories in the family are known.

After launching a few trajectories with initial conditions (3.4), we quickly found that none of the trajectories with initial polar angles $\theta_{i}<25^{\circ}$ return to the vicinity of the nucleus in a time less than $T_{\max }=10 T_{c}$. Therefore we could focus on the region $25^{\circ} \leq \theta_{i} \leq 90^{\circ}$.

We launched 6501 trajectories from $25^{\circ}$ to $90^{\circ}$ ( $\Delta \theta_{i}=0.01$ ) and found a few hundred closed orbits. Of these, it turned out that 65 had an importance number greater than or equal to 3 . We used these 65 orbits in our calculation, and discarded the rest. The mirror images of these 65 orbits through the $\rho$ axis are another set of 65 orbits (except for the one going exactly along the $\rho$ axis). Therefore we have a total of 129 closed orbits in the $m=0$ subspace. The 65 closed orbits with $\theta_{i} \leq 90^{\circ}$ are shown in Fig. 8. Presently we have not found any simple systematic scheme for labeling and characterizing these orbits.

Properties of the most important of these orbits are listed in Table II. The full table is available on request from the authors, and it has been filed with the Physics Auxiliary Publication Service. ${ }^{24}$

For each of the closed orbits found above, we computed the two-dimensional action integral $S_{2}^{k}$ along the trajectory from initial to final spheres, and the twodimensional amplitude factor $A_{2}^{k}$ (this latter quantity was calculated using the method described in an appendix of the accompanying paper). Also, as discussed in paper II, the Maslov index for each orbit was computed by summing the number of extrema in the $\rho$ motion plus the number of crossings of the $z$ axis. The period $T_{k}$ of each closed orbit was also computed. All these results are listed in Table II. (We believe that all quantities are accurate to the number of digits given, except for $A_{2}$, which may have an accuracy of \pm 0.05 , and $N_{\text {imp }}$, which is only semiquantitative by definition.)

Examining this table, we notice that the square root of the importance number is approximately proportional to the semiclassical amplitude factor $A_{2}$. This is no surprise, because both the importance number $N_{\mathrm{imp}}$ and the amplitude factor $A_{2}$ measure the divergence of the family of trajectories around each closed orbit. The importance number is more intuitive and less rigorous, but it is obtained quite trivially from a collection of trajectories. It is helpful in giving a reasonable estimate of the classical amplitude factor $A_{2}$ for each closed orbit (Fig. 9).

We also note a loose connection between the period of the closed orbit and the value of the semiclassical factor $A_{2}$ (Fig. 10). Longer, more complicated orbits usually have smaller values of $A_{2}$. We note also that the shortest, most stable, and most important orbit is the one that goes along the $\rho$ axis; its effect on the spectrum is most prominent, and it was the first to be recognized.

Finally, we see that there are pairs of orbits (Nos. 32 


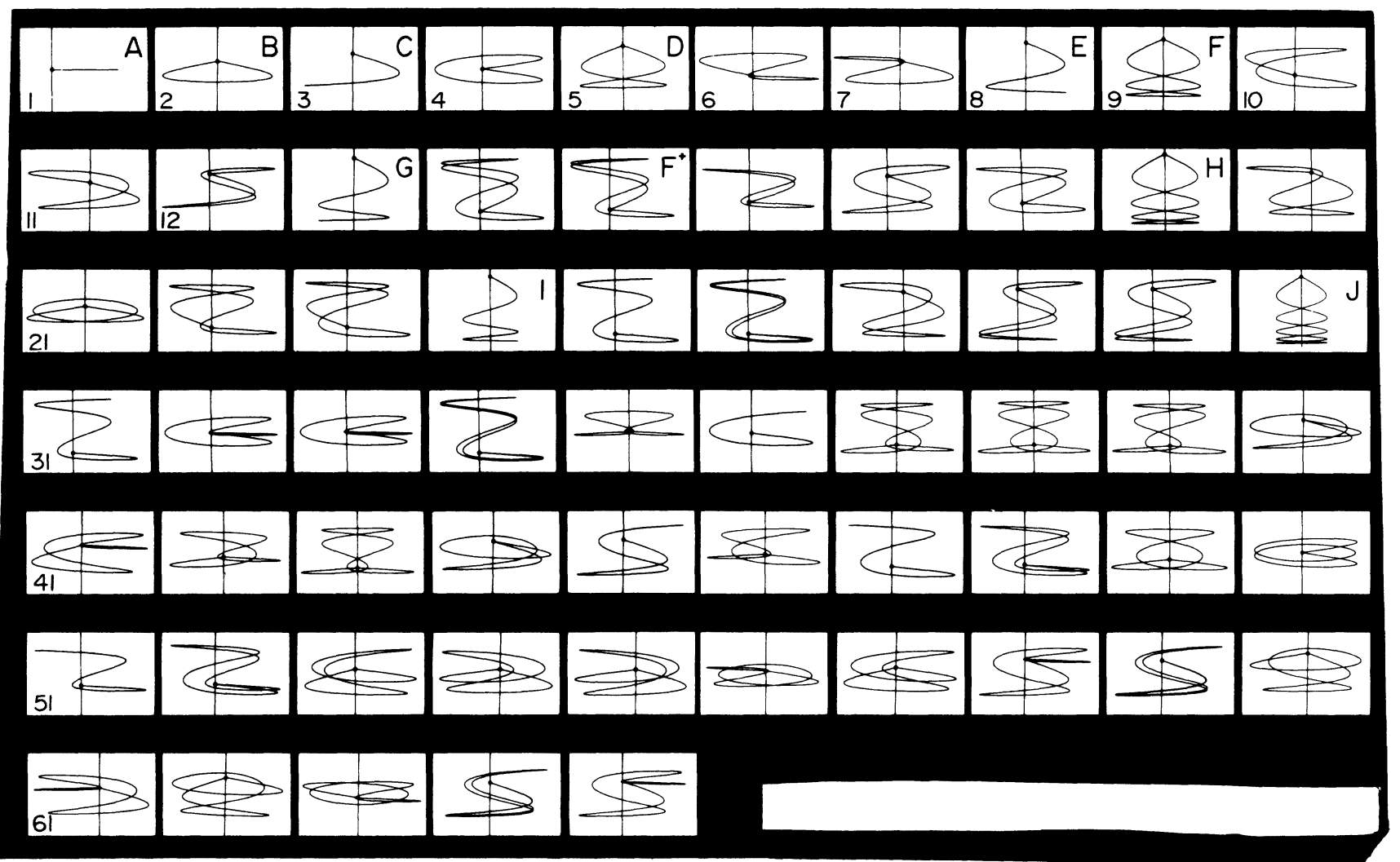

FIG. 8. Important closed orbits for the Hamiltonian, Eq. (1.1).

and 33, for example) having identical oscillation amplitude, phase, and period. In fact, the two orbits are related by time reversal. More generally, any orbit, its timereversed orbit, its mirror image about the $\rho$ axis, and the time-reversed mirror image of the orbit all contribute identical oscillations to the spectrum.

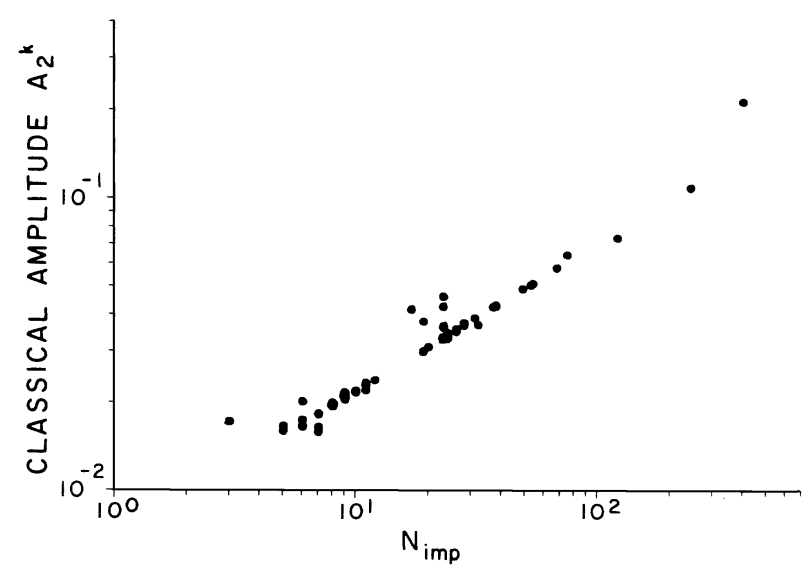

FIG. 9. The (two-dimensional) classical amplitude $A_{2}^{k}$ is plotted vs the importance number $N_{\text {imp }}$ for each orbit. $N_{\text {imp }}$ provides an approximate measure of the classical density, so $A_{2}^{k}$ is approximately proportional to $N_{\mathrm{imp}}^{1 / 2}$.

\section{Spectrum from the closed orbits}

From the preceding information about the closed orbits, and the information previously noted about the initial state and dipole operator, we can compute the absorption spectrum (the average oscillator-strength densi-

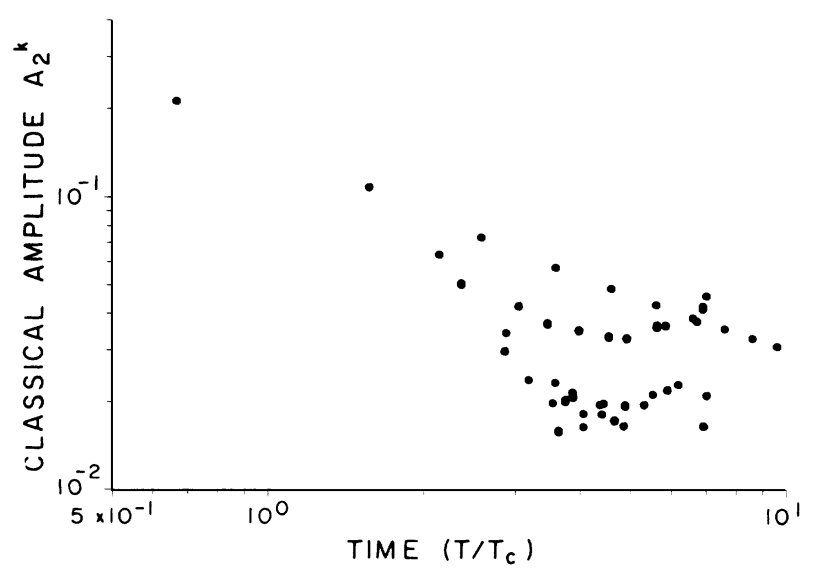

FIG. 10. Classical amplitude is plotted vs the time duration of each orbit. Longer orbits tend to have smaller classical amplitudes, but wide variations exist. 


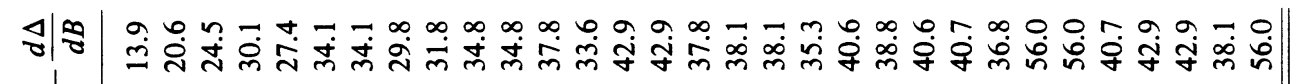

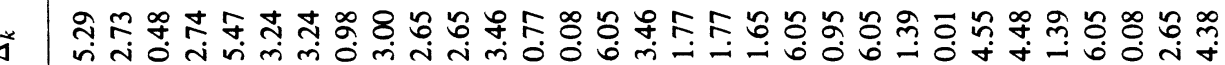

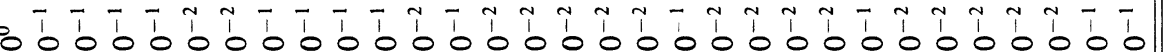

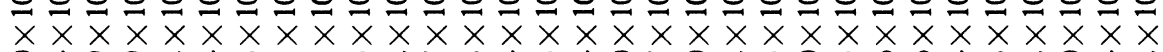

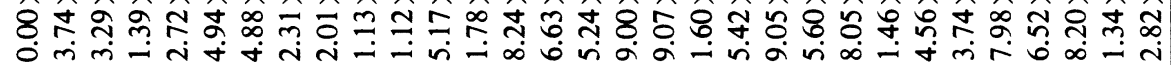

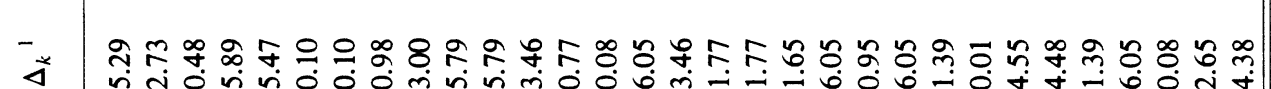

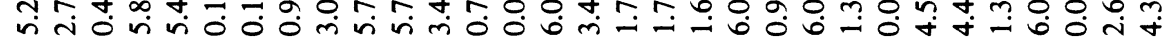

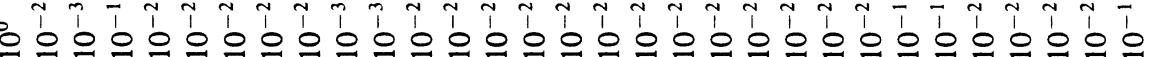

u $\times \times \times \times \times \times \times \times \times \times \times \times \times \times \times \times \times \times \times \times \times \times \times \times \times \times \times \times \times$

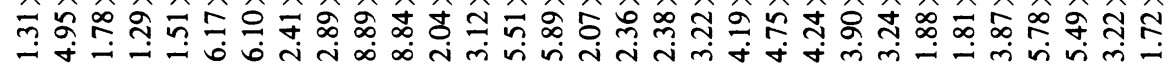

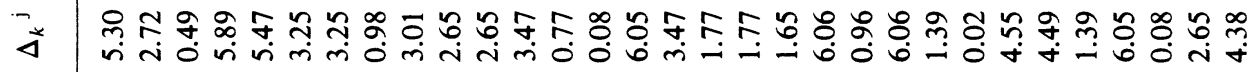

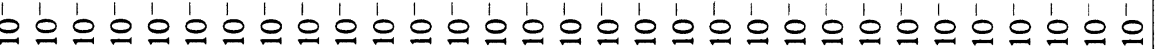
$\times \times \times \times \times \times \times \times \times \times \times \times \times \times \times \times \times \times \times \times \times \times \times \times \times \times \times$

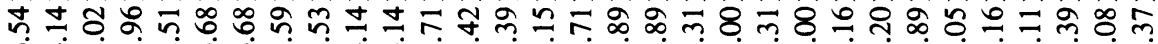

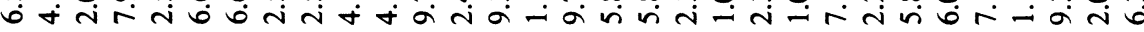

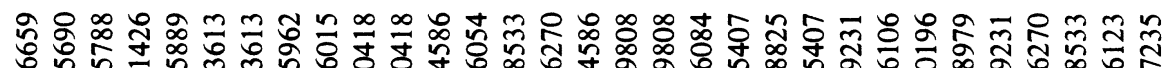
O-

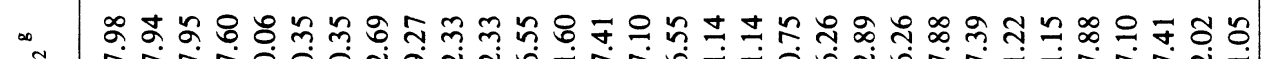

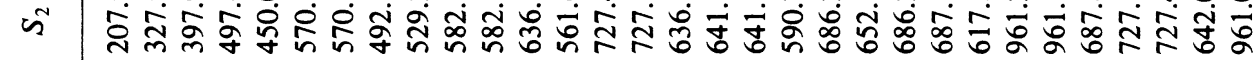

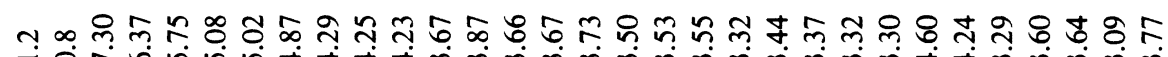

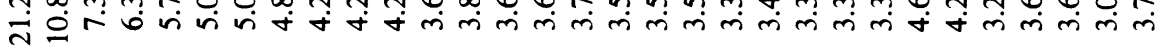

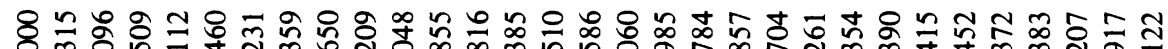

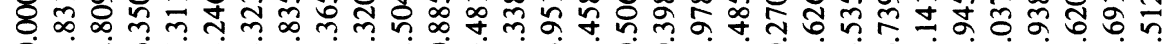

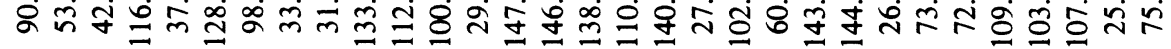


ty) using Eqs. (3.5)-(3.7). The resulting amplitudes and phases of the spectral oscillations are also listed in Table II.

The spectrum $\overline{D f}(E)$ calculated from Eqs. (3.5)-(3.7) (using the 65 most important closed orbits) is shown in Fig. 11. This result is supposed to be compared to the experimentally measured spectrum shown in Fig. 2. However, both theoretical and experimental spectra are so wildly oscillatory that it seems to be impossible to compare them directly.

It is more sensible to compare our calculated ampli- tudes $C_{k}$ and phase $\Delta_{k}$ with the Fourier transform of the absorption spectrum, as computed in Ref. 5(b) and shown in Fig. 3. Let us define

$$
\widetilde{D} f(T)=\int_{E_{1}}^{E_{2}} \overline{D f}(E) \exp (-i T E / \hbar) d E,
$$

where $E_{1}$ and $E_{2}$ are arbitrarily selected limits, close to $E=0$. If we insert Eq. (3.5) into (4.10), neglect the weak dependence of $D f_{0}, C_{k}, T_{k}$, and $\Delta_{k}$ upon $E$, and evaluate the integral, the result is

$$
\begin{aligned}
\widetilde{D} f(T)= & D f(0) \exp \left[-i T\left(E_{1}+E_{2}\right) / 2\right] \frac{\sin \left[T\left(E_{2}-E_{1}\right) / 2\right]}{T / 2} \\
& +\sum_{k} \frac{C_{k} e^{i \Delta_{k}}}{2 i} \exp \left(\frac{-i\left(T-T_{k}\right)\left(E_{2}+E_{1}\right)}{2}\right) \frac{\sin \left[\left(T-T_{k}\right)\left(E_{2}-E_{1}\right) / 2\right]}{\left(T-T_{k}\right) / 2} \\
& -\sum_{k} \frac{C_{k} e^{-i \Delta_{k}}}{2 i} \exp \left(-i \frac{\left(T+T_{k}\right)\left(E_{2}+E_{1}\right)}{2}\right) \frac{\sin \left[\left(T+T_{k}\right)\left(E_{2}-E_{1}\right) / 2\right]}{\left(T+T_{k}\right) / 2}
\end{aligned}
$$

For positive $T$, the second sum can be neglected. Now if $|\widetilde{D} f(T)|^{2}$ is plotted against $T$, distinct peaks should occur at each $T_{k}$, and the height of each peak will be proportional to $\left|C_{k}\right|^{2}$.

Figure 3 shows the square of the Fourier-transformed spectrum, $\tilde{A}(T)$, as calculated in Ref. 5 from the experimental measurements. This experimental result is compared with our theoretical oscillation amplitudes $\left|C_{k}\right|^{2}$ at $E=0$. Because the experimental spectrum is in arbitrary units, the experimental spectrum is normalized so that the highest peak (at $T=0.66 T_{c}$ ) matches the corresponding theoretical one.

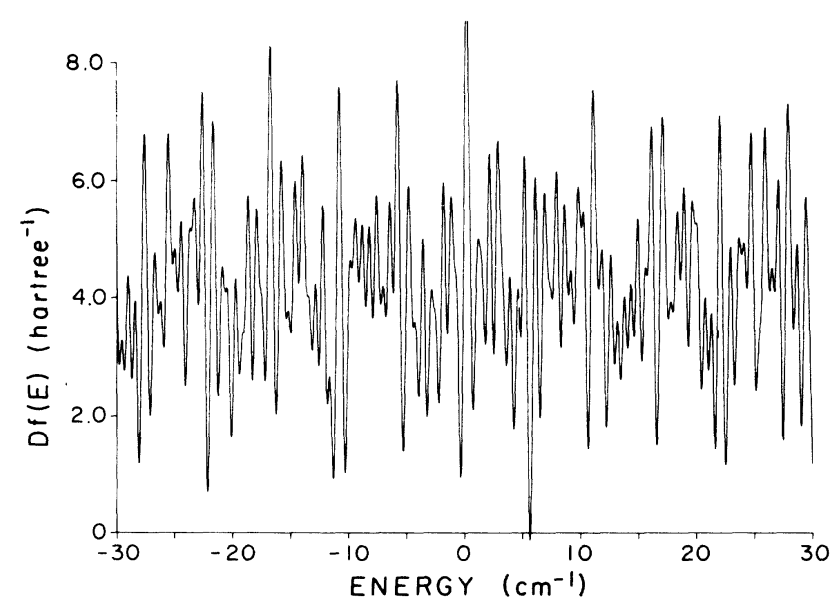

FIG. 11. Computed absorption spectrum $D f(E)$ as a function of energy based upon Eq. (3.5), including the 65 most important closed orbits. Here, as in Fig. 2, peaks and valleys do not correspond to individual states, but to fluctuations associated with the sum of many oscillatory terms [Eq. (3.5)].
The largest and most interesting peaks are arbitrarily labeled $A-J$ and also $A^{\prime}, A^{\prime \prime}$, and $F^{+}$. These are correlated with the closed orbits identified similarly in Fig. 8.

The orbit associated with the peak $A$ is an orbit that goes along the $\rho$ axis. This orbit is the one which Edmonds recognized to be correlated with the "quasiLandau" oscillations observed by Garton and Tomkins. Orbits associated with peaks $B-G$ were found more recently by the Bielefeld University group. ${ }^{5}$

The orbits labeled $A$ and $B$ are classically the two most stable orbits of the system. (Stability is measured by the value of the classical amplitude factor $A_{2}^{k}$; more stable orbits have larger values of $A_{2}^{k}$.) In Table I we find that the classical amplitude factors $A_{2}^{k}$ for orbits 1 and 2 differ by about a factor of 2 . However, their oscillation amplitudes $C_{k}$ differ by an order of magnitude. Why?

This phenomenon is a quantum effect explained intuitively by Reinhardt, and quantitatively by our formulas for $C_{k}$. The outgoing wave $G_{\mathrm{dir}}^{+}\left|D \psi_{i}\right\rangle$ is a combination of an $s$ wave and a $d$ wave $(l=0$ and $l=2)$. The $d$ wave dominates, with an amplitude about four times larger than that of the $s$ wave. Orbit $A$ has its initial and final points at the peak of the $d$ wave $\left(\theta=90^{\circ}\right)$, while orbit $B$ has its initial and final points near the node of the $d$ wave. Therefore the waves propagating outward and back in these two different directions have very different intensities, and their contributions to the spectrum are very different.

The peaks marked $A^{\prime}$ and $A^{\prime \prime}$ come from orbit $A$ repeated once or twice. All of the closed orbits are quite unstable, and each time a pencil of trajectories returns to the vicinity of the nucleus, the trajectories are scattered widely. Hence the classical amplitude $A_{2}^{k}$ and the oscillation amplitude $C_{k}$ associated with two traversals of any closed orbit are much smaller than that for the first 
traversal. Furthermore, amplitudes associated with repeated traversals should decrease in approximately a geometric progression,

$$
C^{A^{\prime \prime}} / C^{A^{\prime}} \simeq C^{A^{\prime}} / C^{A}
$$

The computations confirm this (see Fig. 3).

The peaks marked $F$ and $F^{+}$are interesting, because the corresponding experimental peak at $T=6$ is rather small. The theoretical peak $F$ is associated with a periodic orbit $\left(\theta_{f}=\theta_{i}\right)$ having a rather large amplitude. Peak $\mathrm{F}^{+}$is an orbit of a totally different character: it starts from a different $\theta_{i}$ and follows a totally different path. Note that it is a closed orbit of duration $T$, but not a periodic orbit with that period $-\theta_{f} \neq \theta_{i}$. Each of these two orbits produces a large-amplitude sine-wave oscillation in the spectrum. However, when we look at the absolute phase $\Delta_{k}$ of these two oscillations, we see that they are out of phase by almost exactly $\pi$. Hence for $E$ close to zero, their effects almost cancel.

Overall, the agreement between theory and experiment up to $6 T_{c}$ is very pleasing. Above $6 T_{c}$ the theory predicts additional distinct peaks. The experimental data do not show these peaks. We have no reason to believe that the theory is less accurate for these longer-period orbits, so we tentatively ascribe this discrepancy to problems of experimental resolution: longer period orbits produce very-small-scale fluctuations in the spectrum, which would be difficult to measure accurately.

The theory also gives predictions of the values of the phases $\Delta_{k}$ associated with each oscillation in the spectrum. These phases have not yet been extracted from the experimental data.

\section{B. Other initial states}

We consider now the transitions $2 p_{m= \pm 1} \rightarrow m=0$, $E \simeq 0$ and $2 s \rightarrow m=0, E \simeq 0$. Perusal of Eqs. (3.5) - (3.7) shows that the initial state $\psi_{i}$ enters the formula in only two ways: through the coefficients $b_{l m}^{i}$ in the partialwave expansion of $\left|D \psi_{i}\right\rangle$ and through the radial matrix elements $I\left(n, l, l^{\prime}\right)$. Hence once the information about the classical closed orbits is available, calculation of spectra with various initial states is quite trivial.

We show in Fig. 12 the predicted magnitudes of the spectral-oscillation amplitudes $C_{k}$ for $2 s$ and $2 p_{m= \pm 1}$ initial states. The amplitudes associated with each closed orbit change quite substantially because of the changes in the angular distribution of $G_{E}^{+}\left|D \psi_{i}\right\rangle$ on the initial boundary. For example, when $\psi_{i}$ is the $2 s$ state, then only light linearly polarized along the $z$ axis produces final states with $m=0$. Therefore $D\left|\psi_{i}\right\rangle$ has a nodal surface in the plane $z=0$. As a consequence, the oscillations associated with Edmonds's trajectory $\boldsymbol{A}$ completely disappear. In contrast, the oscillations associated with trajectory $B$ are quite prominent (recall that they were too small to measure when the initial state was $2 p_{z}$ ). Predictions of the phases $\Delta_{k}$ of some of these oscillations are given in Table II.

\section{CONCLUSION}

A theory is now (at last) available which quantitatively accounts for the oscillations observed in the absorption spectrum of a hydrogen atom in a magnetic field. The theory gives information about the finite-resolution spectrum from examination of classical trajectories of the sys-
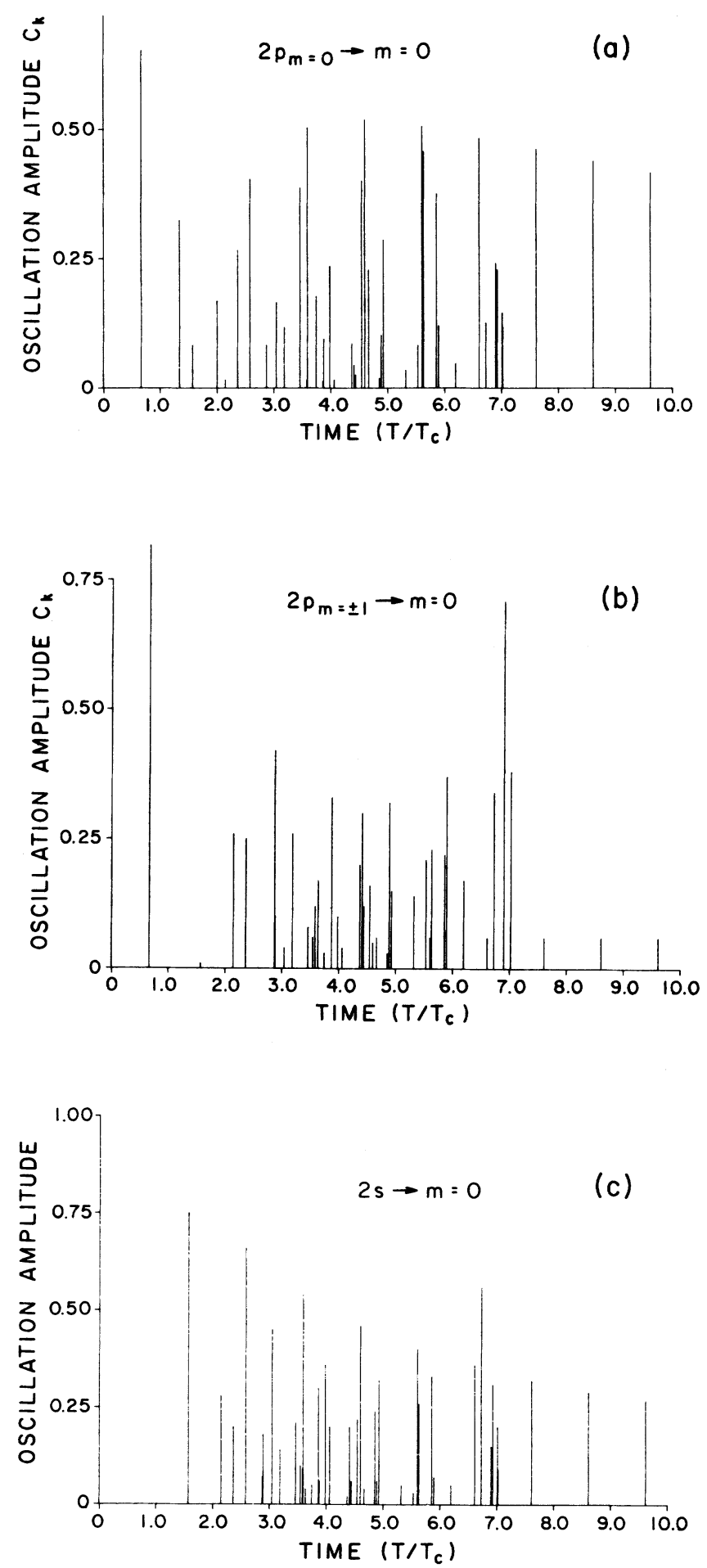

FIG. 12. Predicted spectral oscillation amplitudes $C_{k}$ (not the square) for three initial states. Substantial changes in the patterns are evident. 
tem. (As presently applied, the theory says nothing about individual discrete or quasidiscrete states of the system.)

A more general remark is relevant to the study of quantum properties of classically chaotic systems. Even in such a system, when trajectories are examined on a limited time scale, a kind of order is retained: surrounding each closed orbit is a family of orbits which propagate together for a finite time. Thus finite-time order is manifested in the finite-resolution spectrum: associated with each such family of orbits is a propagating wave which produces interference oscillations that are visible in the absorption spectrum.

In fact, this general principle applies to regular as well as chaotic systems. Whether one looks at the density of states, the average oscillator-strength density, or any other facet of a quantum spectrum, when one examines the quantity at finite resolution, then the quantity will show a smoothly varying background plus a superposition of sinusoidal oscillations; each oscillation will have a wavelength on the energy axis equal to $\lambda_{E}=2 \pi \hbar / T$, where $T$ is the period of a periodic (or possibly a closed) orbit of the corresponding classical system.

\section{ACKNOWLEDGMENTS}

One of us (J.B.D.) acknowledges financial support from the visiting fellowship program of the Joint Institute for Laboratory Astrophysics, National Bureau of Standards and University of Colorado. He gratefully acknowledges the hospitality of the Institute. This work was also supported by the National Science Foundation, through a grant to the College of William and Mary.
*Permanent address.

${ }^{1}$ (a) A review of work on atoms in external fields is given by $\mathbf{C}$. W. Clark, K. T. Lu, and A. F. Starace, in Progress in Atomic Spectroscopy, edited by H. J. Beyer and H. Kleinpoppen (Plenum, New York, 1984), Part C, pp. 247-320; (b) J. B. Delos, S. K. Knudson, and D. W. Noid, Phys. Rev. A 28, 7 (1983); 30, 1208 (1984). Many earlier references are cited therein. See also W. Lamb, Phys. Rev. 85, 259 (1952); R. F. O'Connell, Phys. Lett. 70A, 389 (1979).

${ }^{2}$ W. R. S. Garton and F. S. Tomkins, Astrophys. J. 158, 839 (1969); K. T. Lu, F. S. Tomkins, and W. R. S. Garton, Proc. R. Soc. London, Ser. A 362, 421 (1978).

${ }^{3}$ E. A. Solov'ev, Pis'ma Zh. Eksp. Teor. Fiz. 34, 278 (1981) [JETP Lett. 34, 265 (1981)]; D. R. Herrick, Phys. Rev. A 26, 323 (1982). See also Ref. 1(b).

${ }^{4}$ M. L. Zimmerman, J. C. Castro, and D. Kleppner, Phys. Rev. Lett. 40, 1083 (1978); J. C. Castro, M. L. Zimmerman, R. G. Hulet, D. Kleppner, and R. R. Freeman, ibid. 45, 1780 (1980); D. Kleppner, M. G. Littman, and M. L. Zimmerman, Sci. Am. 244, 130 (1981).

5(a) A. Holle, G. Wiebusch, J. Main, B. Hager, H. Rottke, and K. H. Welge, Phys. Rev. Lett. 56, 2594 (1986); (b) J. Main, G. Wiebusch, A. Holle, and K. H. Welge, ibid. 57, 2789 (1986).

${ }^{6}$ A. R. Edmonds, J. Phys. (Paris) Colloq. 31, C4-71 (1970).

${ }^{7}$ W. P. Reinhardt, J. Phys. B 16, L635 (1983). The wave-packet picture discussed in this paper is most appropriate if a pulsed laser excites the electron to a wave-packet state which is sufficiently compact to give distinct recurrences. In the experiments discussed here, the pulse times are much longer than recurrence times. The physical picture described in the present paper is more consistent with this fact. We consider the steady excitation of electrons into states near the ionization threshold, steady flow of outgoing waves from the vicinity of the nucleus, and steady flow of returning electrons along closed orbits. Interference between the steadily-flowing outgoing and returning waves leads to the oscillations in the spectrum

${ }^{8}$ (a) M. L. Du and J. B. Delos, Phys. Rev. A 38, 1913 (1987) (paper II); (b) brief accounts of this work can be found in M. L. Du and J. B. Delos, Phys. Rev. Lett. 58, 1731 (1987); J. B. Delos and M. L. Du, IEEE J. Quant. Electron. (to be pub- lished); J. B. Delos and M. L. Du, in Atomic Spectra and Collisions in External Fields 2, edited by K. T. Taylor (Plenum, New York, 1987); (c) A different approach to this problem is presented by D. Wintgen, Phys. Rev. Lett. 58, 1589 (1987); D. Wintgen and H. Friedrich, Phys. Rev. A 36, 131 (1987).

${ }^{9}$ The historical development of Hamilton's analogy between wave motion and particle motion is presented by T. L. Hankins, Sir William Rowan Hamilton (Johns Hopkins University Press, Baltimore, 1980).

${ }^{10}$ The correspondence principle is expressed in modern mathematical language by V. P. Maslov and M. V. Fedoriuk, Semiclassical Approximation in Quantum Mechanics (Reidel, Boston, 1981).

${ }^{11} \mathrm{~A}$ simplified presentation of the ideas in Ref. 10 is given by $\mathrm{J}$. B. Delos, Adv. Chem. Phys. 65, 161 (1986); S. K. Knudson, J. B. Delos, and B. Bloom, J. Chem. Phys. 83, 5703 (1985); S. K. Knudson, J. B. Delos, and D. W. Noid, ibid. 84, 6886 (1986); see also J. B. Delos, ibid. 86, 425 (1987).

${ }^{12}$ (a) A. R. P. Rau, Comments At. Mol. Phys. 10, 19 (1980); (b) A. F. Starace, J. Phys. B 6, 585 (1973); (c) M. A. Al-Laithy, P. F. O'Mahony, and K. T. Taylor, ibid. 19, L773 (1986).

${ }^{13}$ U. Fano [J. Phys. B 13, L519 (1980); Phys. Rev. A 22, 2660 (1980)] discussed the Garton-Tomkins oscillations in terms of motion on a potential-energy ridge. While these ideas are very stimulating, more recent experiments show that observed oscillations are correlated with closed orbits, most of which have no relationship to the potential-energy ridge.

${ }^{14}$ (a) A. Einstein, Verh. Dtsch. Phys. Ges. 19, 82 (1917); (b) I. C. Percival, Adv. Chem. Phys. 36, 1 (1977).

${ }^{15} \mathrm{M}$. V. Berry, in The Wave-Particle Dualism, edited by S. Diner et al. (Reidel, Boston, 1984), pp. 231-252; in Chaotic Behavior of Deterministic Systems, Les Houches Session XXXVI, edited by G. Ioos et al. (North-Holland, Amsterdam, 1983), pp. 171-272.

${ }^{16}$ M. C. Gutzwiller, J. Math. Phys. 8, 1979 (1967); 10, 1004 (1969); 11, 1791 (1970); 12, 343 (1971).

${ }^{17}$ R. Balian and C. Bloch, Ann. Phys. (N.Y.) 60, 401 (1970); 64, 271 (1971); 69, 76 (1972); 85, 514 (1974).

${ }^{18}$ M. V. Berry and M. Tabor, Proc. R. Soc. London, Ser. A 349, 101 (1976).

${ }^{19}$ M. V. Berry, Ann. Phys. (N.Y.) 131, 163 (1981). 
${ }^{20}$ There is a subtlety here. Equation (2.4) is presumed to be valid in the limit $\hbar \rightarrow 0$ for those paths that travel from $\mathbf{q}^{\prime}$ to $\mathbf{q}$ within a finite time $T$. However, in a bounded system, an infinite number of distinct trajectories travel from $\mathbf{q}^{\prime}$ to $\mathbf{q}$, and there is no upper limit on the travel time. To obtain a spectrum to arbitrary resolution from closed orbits, we would have to apply Eq. (2.4) for fixed $\hbar$ in the limit $T \rightarrow \infty$. We do not know whether the formula is applicable in that limit.

${ }^{21}$ Obviously, a wave is correlated not with any single closed orbit by itself, but rather with the family or pencil of orbits surrounding the closed orbit.

${ }^{22}$ H. A. Bethe and E. E. Salpeter, Quantum Mechanics of Oneand Two-Electron Atoms (Springer, New York, 1957).

${ }^{23}$ The importance number approximately measures the classical density of returning trajectories. (This is like estimating local magnetic field strength by counting field lines entering a sur- face.) The number is useful because it is found immediately from the trajectory integration; evaluation of the classical density takes more work.

${ }^{24}$ See AIP document No. PAPS PLRAA-38-1896-3 for 3 pages of Table II. Order by PAPS number and journal reference from American Institute of Physics, Auxiliary Publication Service, 335 East 45th Street, New York, NY 10017. The prices are $\$ 1.50$ for microfiche and $\$ 5$ for photocopies. Airmail additional. Make checks payable to the American Institute of Physics.

${ }^{25}$ There are two differences between the present theoretical result and that reported in Ref. 8(a). (i) We now include amplitudes for orbits $A^{\prime}$ and $A^{\prime \prime}$, which we did not include previously. (ii) The previously reported amplitudes were a factor of 2 too large; we had incorrectly arrived at a factor $2^{23 / 4}$ instead of $2^{19 / 4}$ in Eq. (3.7a). 

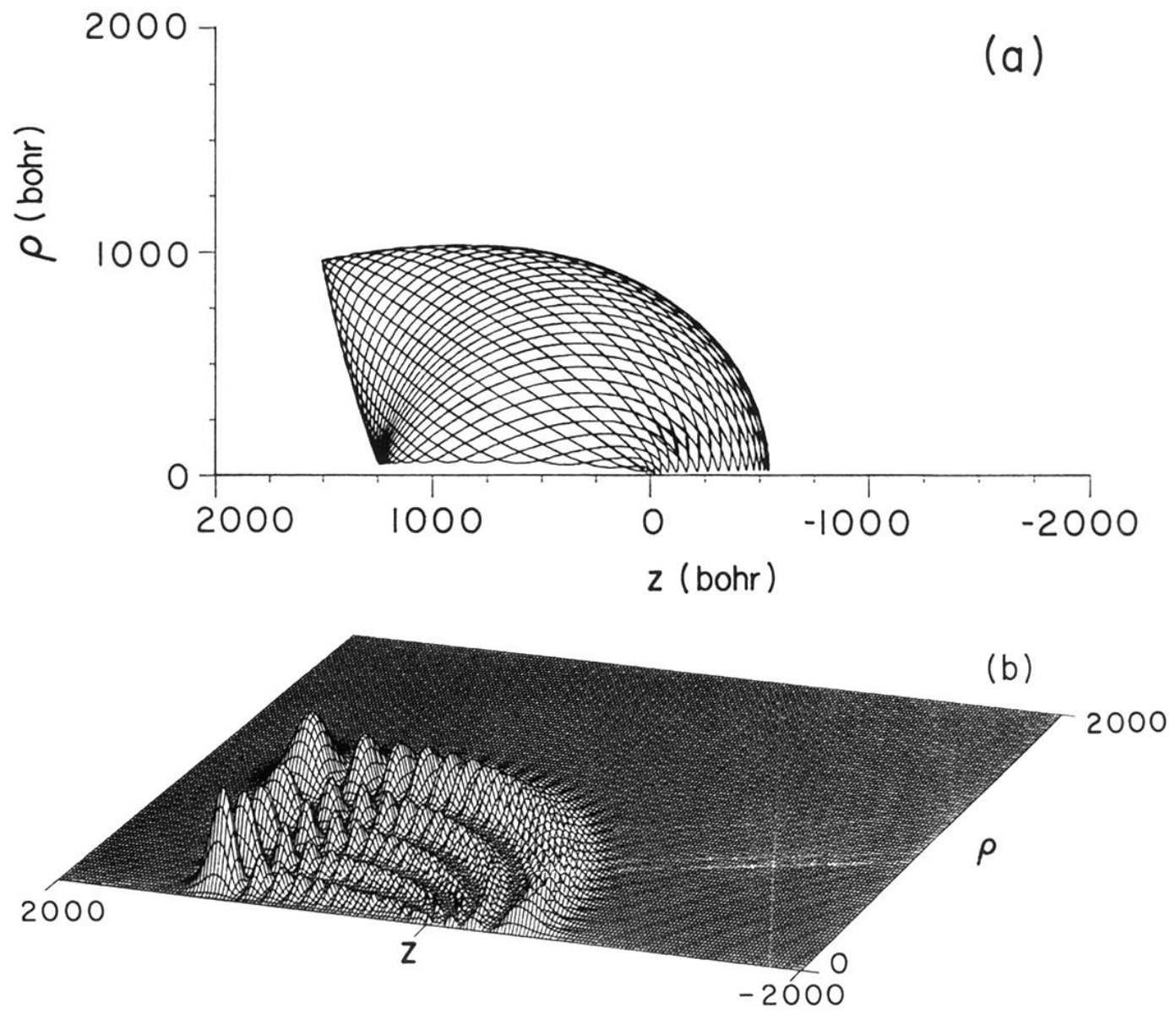

FIG. 4. (a) Regular trajectory of an atomic electron in Coulomb and diamagnetic fields (there is also a weak electric field pointing in the $-z$ direction, but it has little effect). The path followed by the electron is represented in cylindrical coordinates. This trajectory is one of the typical types that are associated with the regular part of the spectrum $(n \simeq 30, B \sim$ a few teslas). The momentum field has four branches. Associated with this momentum field is a semiclassical wave function given by Eq. (2.2b). (b) Quantum wave function correlated with the trajectory shown in Fig. 4(a). The wave function is large and oscillatory in the region of $(\rho, z)$ space occupied by the trajectory, and exponentially small in the rest of space. The number of oscillations in the wave function is correlated with classical action integrals. This wave function was calculated directly from quantum mechanics. The semiclassical approximation discussed in the text would give almost exactly the same result. 


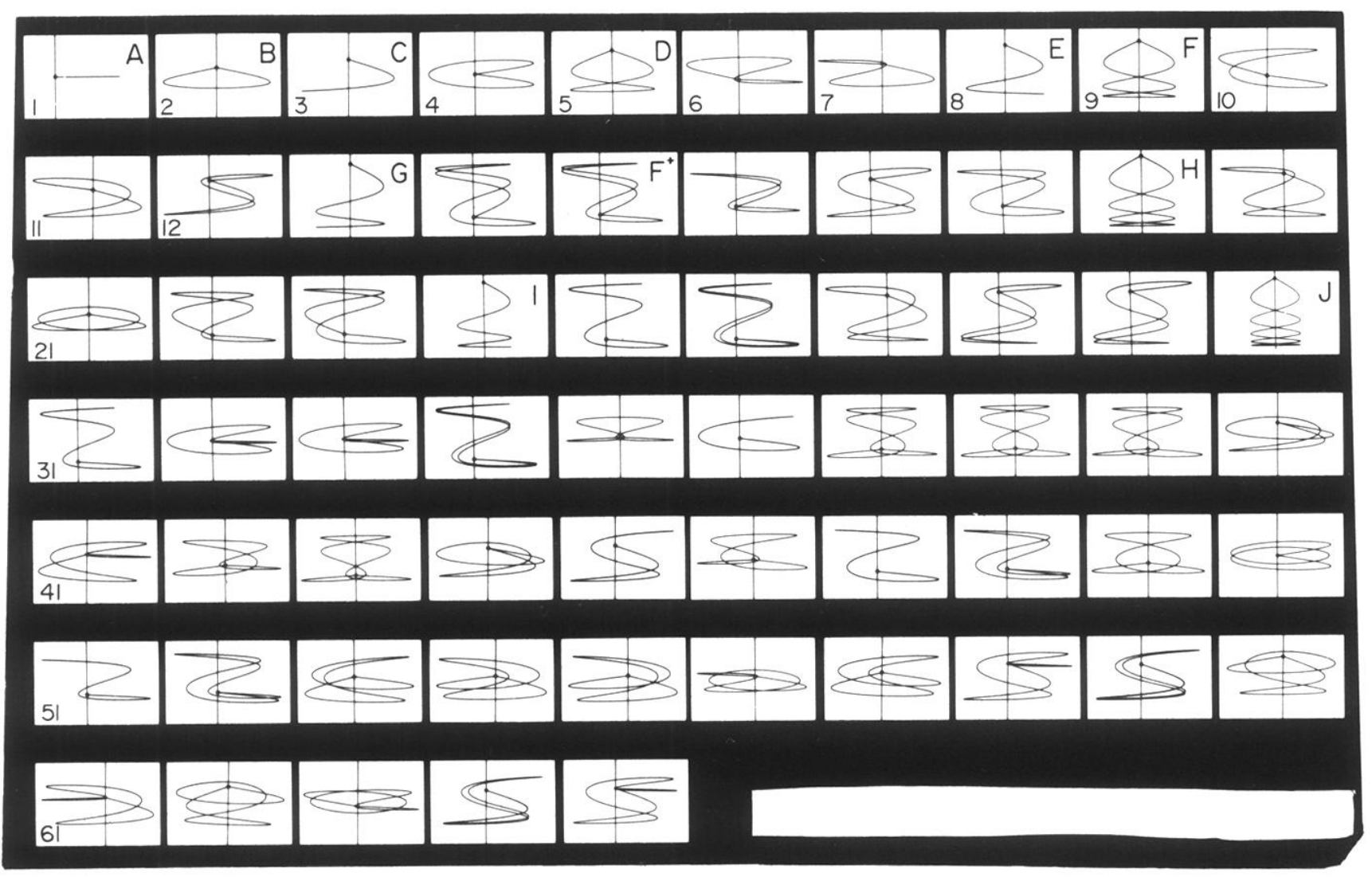

FIG. 8. Important closed orbits for the Hamiltonian, Eq. (1.1). 